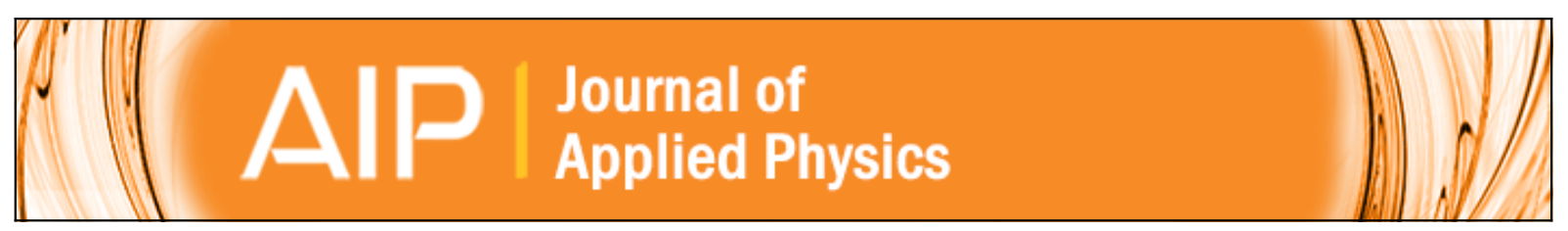

\title{
A semi-analytical model for semiconductor solar cells
}

D. Ding, S. R. Johnson, S.-Q. Yu, S.-N. Wu, and Y.-H. Zhang

Citation: Journal of Applied Physics 110, 123104 (2011); doi: 10.1063/1.3671061

View online: http://dx.doi.org/10.1063/1.3671061

View Table of Contents: http://scitation.aip.org/content/aip/journal/jap/110/12?ver=pdfcov

Published by the AIP Publishing

\section{Articles you may be interested in}

Ultra-thin GaAs single-junction solar cells integrated with a reflective back scattering layer

J. Appl. Phys. 115, 203105 (2014); 10.1063/1.4878156

The effect of mode excitations on the absorption enhancement for silicon thin film solar cells

J. Appl. Phys. 114, 233104 (2013); 10.1063/1.4851817

Relationship between the cell thickness and the optimum period of textured back reflectors in thin-film microcrystalline silicon solar cells

Appl. Phys. Lett. 102, 053509 (2013); 10.1063/1.4790642

Analyzing nanotextured transparent conductive oxides for efficient light trapping in silicon thin film solar cells Appl. Phys. Lett. 101, 103903 (2012); 10.1063/1.4750242

Reduction of crosshatch roughness and threading dislocation density in metamorphic GalnP buffers and GalnAs solar cells

J. Appl. Phys. 111, 103528 (2012); 10.1063/1.4721367

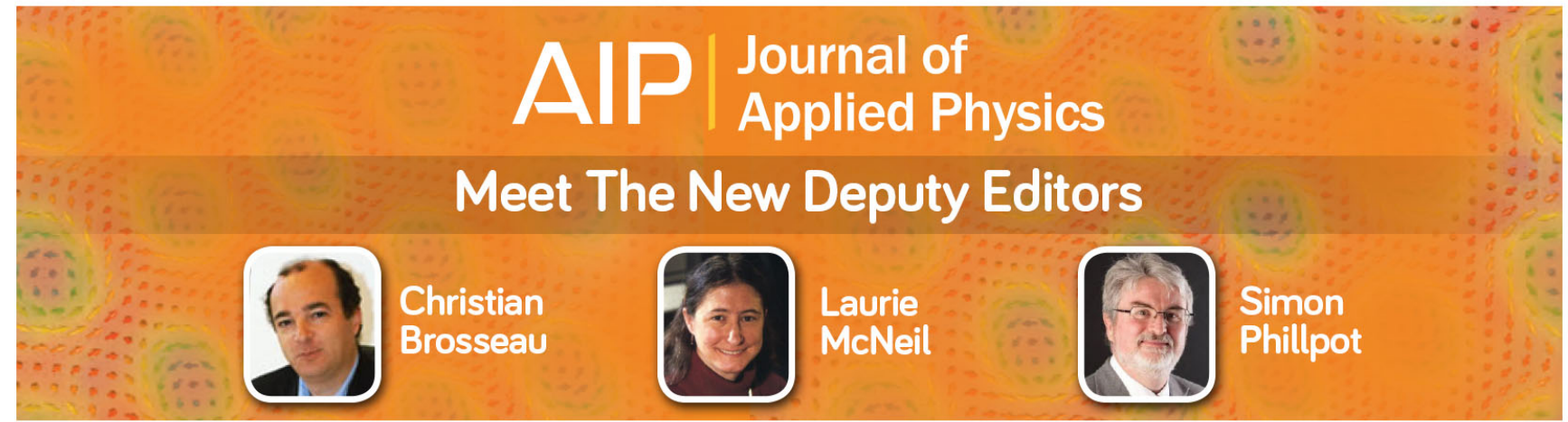




\title{
A semi-analytical model for semiconductor solar cells
}

\author{
D. Ding, S. R. Johnson, S.-Q. Yu, ${ }^{\text {a) }}$ S.-N. Wu, ${ }^{\text {b) }}$ and Y.-H. Zhang ${ }^{\text {() }}$ \\ Center for Photonics Innovation and School of Electrical, Computer, and Energy Engineering, \\ Arizona State University, Tempe, Arizona 85287, USA
}

(Received 4 August 2011; accepted 15 November 2011; published online 23 December 2011)

\begin{abstract}
A semi-analytical model is constructed for single- and multi-junction solar cells. This model incorporates the key performance aspects of practical devices, including nonradiative recombination, photon recycling within a given junction, spontaneous emission coupling between junctions, and non-step-like absorptance and emittance with below-bandgap tail absorption. Four typical planar structures with the combinations of a smooth/textured top surface and an absorbing/reflecting substrate (or backside surface) are investigated, through which the extracted power and four types of fundamental loss mechanisms, transmission, thermalization, spatial-relaxation, and recombination loss are analyzed for both single- and multi-junction solar cells. The below-bandgap tail absorption increases the short-circuit current but decreases the output and open-circuit voltage. Using a straightforward formulism this model provides the initial design parameters and the achievable efficiencies for both single- and multiple-junction solar cells over a wide range of material quality. The achievable efficiency limits calculated using the best reported materials and AM1.5 G one sun for GaAs and Si single-junction solar cells are, respectively, 27.4 and $21.1 \%$ for semiconductor slabs with a flat surface and a non-reflecting index-matched absorbing substrate, and 30.8 and $26.4 \%$ for semiconductor slabs with a textured surface and an ideal $100 \%$ reflecting backside surface. Two important design rules for both single- and multi-junction solar cells are established: i) the optimal junction thickness decreases and the optimal bandgap energy increases when nonradiative recombination increases; and ii) the optimal junction thickness increases and the optimal bandgap energy decreases for higher solar concentrations. (C) 2011 American Institute of Physics. [doi:10.1063/1.3671061]
\end{abstract}

\section{INTRODUCTION}

Photovoltaic solar cell modeling is often approached in two different directions: i) numerical simulations based on drift and diffusion models, ${ }^{1}$ and ii) analytical analysis based on detailed balance models. ${ }^{2,3}$ Although commercial simulation software can predict a reasonable performance for a given design, complex simulations consume a large amount of computation time and it is not straightforward to extract clear device physics from complicated numerical calculations, especially for multi-junction solar cells. Moreover, most commercial software packages consider both radiative and non-radiative recombination ${ }^{4-6}$ however, they typically do not include some important aspects related to radiative recombination, such as photon recycling ${ }^{7,8}$ within a given junction and spontaneous emission coupling between adjacent junctions. ${ }^{9-12}$ On the contrary, detailed balance models are only capable of clarifying the fundamental limitations of ideal solar cells by neglecting many important mechanisms that occur in real devices. It is, therefore, necessary to develop a semi-analytical model that not only takes into

\footnotetext{
${ }^{a)}$ Present address: Department of Electrical Engineering, University of Arkansas, Fayetteville, Arkansas 72701, USA.

b) Present address: Philips Lumileds Lighting Company, San Jose, California 95131, USA.

${ }^{c}$ Author to whom correspondence should be addressed. Electronic mail: yhzhang@asu.edu.
}

account all of the important properties of materials and device structures to provide a more thorough understanding of photovoltaic solar cells, but also enables fast computation to offer basic guidance for practical device design. The development of such a model has been carried out and some of the key results were briefly reported in Ref. 13.

This paper reports the detailed work that examines the detailed balance theories and extends them in the construction of a semi-analytical model for single- and multijunction solar cells, which explicitly elucidates the impact of real material and device properties, including nonradiative recombination, photon recycling, spontaneous emission coupling, and the non-step-like absorptance and emittance of junctions and materials with absorption tails below the bandgap. As a result, this model provides: i) an in-depth analysis of radiative losses, ii) a straightforward formulism compared to drift and diffusion models, and iii) important optimal design parameters, such as bandgap energy, junction thickness, and junction number, over a wide range of material quality and solar concentration.

\section{FUNDAMENTAL LOSS MECHANISMS IN SOLAR CELLS}

It is imperative to identify the loss mechanisms in conventional $p-n$ junction solar cells and to establish which of these limit efficiency. For example, the loss mechanisms for incident solar photons include reflection, contact shadowing, 
absorption in barrier layers and by free-carriers, and transmission through the junction. Of these losses, reflection can be significantly reduced using anti-refection coatings ${ }^{14}$ and textured surfaces, ${ }^{4,15}$ contact shadowing can be minimized or eliminated by using transparent ${ }^{16}$ or backside contacts, ${ }^{17}$ and parasitic absorption can be minimized using widebandgap barriers and optimal doping profiles in the junction. Conversely, transmission losses are inherent to photovoltaic solar cells and strongly depend on the optical properties of the junction materials.

The loss mechanisms related to photogenerated carriers include: i) thermalization of carriers from their initial excited states to the band edges, ii) spatial relaxation of carriers as they are swept along the band-edges to the contacts, iii) radiative recombination of electron-hole pairs, iv) ShockleyRead-Hall (SRH), Auger, and surface recombination, ${ }^{6}$ and v) series and shunt resistance. Of these losses, surface recombination is structure dependent and can be significantly reduced using wide-bandgap barriers, the series resistance can be minimized using well designed junctions and contacts, and shunt resistance can be minimized using high quality single-crystal materials and optimized fabrication techniques. ${ }^{18}$ Therefore, without losing generality, this study focuses on all of the fundamental loss mechanisms and neglects surface recombination and series and shunt resistance.

The band edge diagram of the single-junction heterostructure solar cell shown in Fig. 1 schematically illustrates the key loss mechanisms. The solar radiation transmitted through the junction at energies below the optical bandgap is the major component of the transmission loss. The solar radiation absorbed in the junction generates excess carriers that thermally relax to the band-edges (almost instantaneously) in a loss process called thermalization. As the carriers are swept to the junction contacts, they lose potential energy as they move along the band edge in a loss mechanism referred to as spatial relaxation in this work. Before the electrons and holes are extracted from the junction contacts, some are lost to radiative and nonradiative recombination.

To clearly demonstrate the impact of these loss mechanisms on the performance of single-junction solar cells, two characteristic current-voltage curves are shown in Fig. 2; Fig. 2(a) is the ideal case where only radiative recombination is present, and Fig. 2(b) is the non-ideal case where both

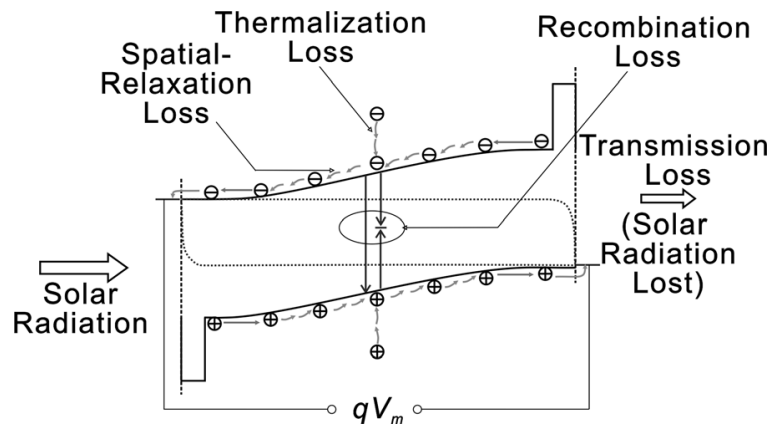

FIG. 1. Schematic band-edge diagram showing the key loss mechanisms for a typical solar cell.
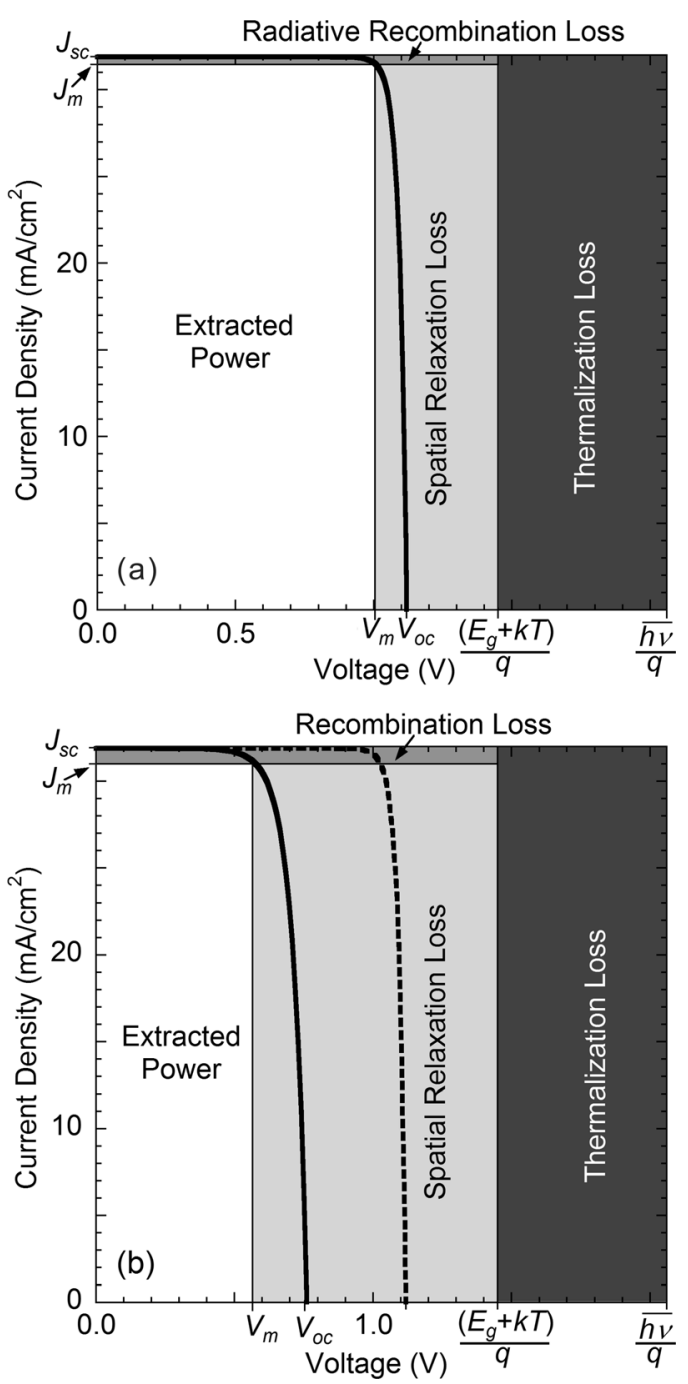

FIG. 2. Current vs voltage graph for (a) an ideal GaAs solar cell without nonradiative recombination losses, and (b) a non-ideal GaAs solar cell with nonradiative recombination losses. The area of the various shaded regions represents the power extracted and lost. Also shown are the short-circuit current, $J_{s c}$, operating current, $J_{m}$, average absorbed solar photon energy, $\overline{h \nu}$, bandgap plus thermal energy, $E_{g}+k T$, open-circuit voltage, $V_{o c}$, and operating voltage, $V_{m}$.

radiative and nonradiative recombination are present. Here, the material properties of GaAs and the AM1.5 G solar spectrum $^{19}$ are used. The equations utilized in the calculation are presented in the following sections. A relatively large nonradiative recombination rate (representing poor quality material) is used in Fig. 2(b) to clearly illustrate the differences between the two cases. Furthermore, the amount of incident solar power that is extracted and lost is quantified by the areas of the current-voltage rectangles that are labeled accordingly. This diagram shows both the relative contribution of each loss mechanism and the relative interplay between the loss mechanisms. Although the power lost directly to carrier recombination is not significantly increased, the impact on spatial relaxation is substantial; this is a result of the large internal electrical field required to rapidly sweep carriers out of the junction in order to limit the otherwise large and ever-present carrier recombination losses. Note that the trade-off between recombination and 
spatial-relaxation losses also occurs during the presence of other loss mechanisms such as surface recombination and series and shunt resistance.

Also shown in Fig. 2 are the short-circuit current, $J_{s c}$, the open-circuit voltage, $V_{o c}$, the current, $J_{m}$, and voltage, $V_{m}$, at maximum power output, ${ }^{1,6,18}$ the average energy of the absorbed solar photons, $\overline{h v}$, the average energy separation of the thermalized electron and hole populations $\left(E_{g}+k T\right)$, and the electron charge, $q$. The recombination current loss is $\left(J_{s c}-J_{m}\right)\left(E_{g}+k T\right) / q$, the spatial-relaxation loss is $J_{m}\left(E_{g}+k T-q V_{m}\right) / q$, and the thermalization loss is $J_{s c}\left[\overline{h \nu}-\left(E_{g}+k T\right)\right] / q$, which is the same for both cases in Fig. 2, and the total amount of solar power absorbed is $J_{s c} \overline{h \nu} / q$, assuming $100 \%$ internal quantum efficiency under the short-circuit condition. ${ }^{18}$ These examples are considered under the optimal working conditions; however, the principle previously discussed and shown in the figures can also be applied to other operation points.

\section{A SEMI-ANALYTICAL MODEL FOR SOLAR CELLS}

\section{A. Assumptions and basic formula}

As pointed out by Henry, ${ }^{3}$ in addition to nonradiative recombination, detailed balance models ignore many of the extrinsic losses in practical solar cells such as reflection, contact shadowing, series and shunt resistance, incomplete collection of photogenerated carriers, absorption in window layers, and a rise in junction temperature. Other assumptions commonly made that are not explicitly stated are absorbing substrates, step-like absorbance and emittance (i.e., absorbing layers are assumed to be opaque at energies above the bandgap and transparent at energies below the bandgap), the Boltzmann approximation for photon and carrier statistics, constant refractive index, and unity internal quantum efficiency. ${ }^{18}$ These assumptions are carefully examined and clarified in this work.

The current density of a single-junction solar cell is,

$$
J=J_{s c}-\left(J_{\text {rad }}+J_{S R H}+J_{\text {Auger }}\right),
$$

where $J$ is the total current density, $J_{s c}$ is the short-circuit current density, $J_{\text {rad }}$ is the radiative recombination current density, $J_{S R H}$ is the SRH recombination current density, and $J_{\text {Auger }}$ is the Auger recombination current density. Equation (1) is the basic formula for the model and the analysis of each term is provided in the following sections.

\section{B. Short-circuit current density}

The short-circuit current density is the rate at which photogenerated carriers are collected under the short-circuit condition. Taking into account the absorption within the width of the Urbach tail, ${ }^{20}$ the short-circuit current density per unit area is,

$$
J_{s c}=q \int_{0}^{\infty} A_{\alpha} \eta_{i} n_{s u n} \times d h \nu=q \overline{A_{\alpha}} \int_{E_{g}-E_{u}}^{\infty} n_{\text {sun }} \times d h \nu,
$$

where $q$ is the electron charge, $A_{\alpha}$ is the energy-dependent absorptance of the junction, $\eta_{i}$ is the energy-dependent inter- nal quantum efficiency (i.e., capture efficiency), ${ }^{16} E_{g}$ is the bandgap energy, $E_{u}$ is the width of the Urbach tail, and $n_{\text {sun }}$ is the solar photon flux density per unit area. For simplicity, $\eta_{i}=1$ is assumed, which is valid since, typically, a very large fraction of the photogenerated carriers are collected under the short-circuit condition. The $E_{g}-E_{u}$ term represents an effective bandgap cut-off that is $E_{u}$ smaller than $E_{g}$ when the influence of localized tail states is considered.

The absorptance term is moved outside the integral in Eq. (2) by defining the energy independent effective absorptance as,

$$
\overline{A_{\alpha}} \equiv \frac{\int_{0}^{\infty} A_{\alpha} n_{\text {sun }} \times d h \nu}{\int_{E_{g}-E_{u}}^{\infty} n_{\text {sun }} \times d h \nu} .
$$

Here, the effective absorptance represents a convenient measure of the "optical thickness" of a junction.

The concept of below-bandgap absorption with a characteristic width can be extended to include other extrinsic mechanisms such as impurity bands, quantum wells, quantum wires, and quantum dots which add localized energy states below the bandgap. The details can be slightly different but the basic principle remains the same in that the effective bandgap shifts to a lower energy as the width of the tail increases which results in an increase in the short-circuit current and a reduction in the open-circuit voltage.

\section{Radiative recombination current density}

Radiative recombination is one of the intrinsic loss mechanisms in solar cells. Photons generated through radiative recombination typically undergo many absorption/emission cycles before escaping. This process is commonly referred as photon recycling ${ }^{7,8}$ and is not negligible in optically-thick semiconductor devices such as solar cells. Taking photon recycling into account, the radiative recombination current density is,

$$
\begin{aligned}
J_{\text {rad }} & =q d\left(1-\gamma_{r}\right) R_{s p}=q d\left(\gamma_{e, \text { upper }}+\gamma_{e, \text { lower }}\right) R_{s p} \\
& =q\left(N_{s p, \text { upper }}+N_{s p, \text { lower }}\right)
\end{aligned}
$$

where $d$ is the junction thickness, $\gamma_{r}$ is the photon recycling factor, ${ }^{8}$ and $R_{s p}$ is the radiative recombination rate per unit area per unit length. Furthermore, assuming that the parasitic absorption of spontaneous emission due to impurities and free carriers is negligible, then $\left(1-\gamma_{r}\right)=\gamma_{e}$, where $\gamma_{e}$ is the photon extraction factor. ${ }^{8}$ Moreover, photon extraction factors for both the upper and the lower surfaces are specified, since the upper and lower surface configurations of a given junction are different. In the right-most equation, the radiative recombination current is given in terms of $N_{s p, u p p e r}$ and $N_{s p \text {,lower }}$, which provide the spontaneous emission extracted through the respective upper and lower surfaces of the junction.

Taking ambient blackbody radiation into account, the net spontaneous emission fluxes from the upper surface into free space and from the lower surface into a similar semiconductor material are, respectively, 


$$
\begin{aligned}
N_{s p, u p p e r}= & \int_{0}^{\infty} n_{\text {sp }, \text { upper }} \times d h \nu \\
= & \frac{2 \pi}{h^{3} c^{2}} \int_{0}^{\infty} \varepsilon_{\text {upper }}\left(n_{\nu, s p}-n_{\nu, b b}\right)(h \nu)^{2} d h \nu \\
= & \frac{2 \pi}{h^{3} c^{2}} \bar{\varepsilon}_{\text {upper }} \int_{E_{g}-E_{u}}^{\infty}\left(n_{\nu, s p}-n_{\nu, b b}\right)(h \nu)^{2} d h \nu \\
\cong & \frac{2 \pi}{h^{3} c^{2}} \bar{\varepsilon}_{\text {upper }} k T\left(E_{g}-E_{u}+k T\right)^{2} e^{-\left(E_{g}-E_{u}\right) / k T} \\
& \times\left(e^{q V / k T}-1\right), \\
N_{s p, l o w e r}= & \int_{0}^{\infty} n_{s p, l o w e r} d h \nu \\
= & \frac{2 \pi}{h^{3} c^{2}} \int_{0}^{\infty} n_{r}^{2} \varepsilon_{\text {lower }}\left(n_{\nu, s p}-n_{\nu, b b}\right)(h \nu)^{2} d h \nu \\
= & \frac{2 \pi n_{g}^{2}}{h^{3} c^{2}} \bar{\varepsilon}_{\text {lower }} \int_{E_{g}-E_{u}}^{\infty}\left(n_{\nu, s p}-n_{\nu, b b}\right)(h \nu)^{2} d h \nu \\
\cong & \frac{2 \pi n_{g}^{2}}{h^{3} c^{2}} \bar{\varepsilon}_{\text {lower }} k T\left(E_{g}-E_{u}+k T\right)^{2} e^{-\left(E_{g}-E_{u}\right) / k T} \\
& \times\left(e^{q V / k T}-1\right),
\end{aligned}
$$

where $n_{s p, u p p e r}$ and $n_{s p, l o w e r}$ are the spontaneous emission fluxes per unit energy interval from the upper and lower surfaces, respectively, $h$ is Planck's constant, $c$ is the speed of light in vacuum, $k$ is Boltzmann's constant, $n_{r}$ is the refractive index, $h \nu$ is the photon energy, and the emittance is $\varepsilon_{\text {upper }}$ for the upper surface and $\varepsilon_{\text {lower }}$ for the lower surface. The respective photon occupation numbers for the spontaneous emission and blackbody background are $n_{\nu, s p}=\left[e^{(h \nu-q V) / k T}-1\right]^{-1}$ and $n_{\nu, b b}=\left[e^{h \nu / k T}-1\right]^{-1}$, where $V$ is the output voltage, assuming $q V$ is equal to the separation between the electron and hole quasi-Fermi levels in the junction (which implies no series or contact resistance).

Equation (5) implies that the absorptance of the junction, in terms of ambient blackbody radiation, is equal to the emittance of the junction, in terms of spontaneous emission, which is a reasonable assumption when the internal optical loss is negligible. ${ }^{21}$ Note that the spectral spontaneous emission from the upper surface, $n_{s p, u p p e r}$, is experimentally measurable, however, the information it provides must be carefully interpreted. From Eq. (5a), $n_{s p, \text { upper }}$ is clearly a function of temperature, bandgap, tail width, emittance of upper surface, and injection level, which is $q V$ in this study, however, more precisely, it is the quasi Fermi-level separation of electrons and holes. Parameters such as capture efficiency influence the injection level, however, $n_{s p \text {,upper }}$ is not a direct measure of these.

The refractive index is a slowly varying function compared to either the sharp cutoff of the tail states or the occupation number and is therefore moved out from the integral of Eq. (5b), by defining an average value as,

$$
n_{g}^{2} \equiv \frac{\int_{0}^{\infty} n_{r}^{2} \varepsilon_{\text {lower }}\left(n_{\nu, s p}-n_{\nu, b b}\right)(h \nu)^{2} d h \nu}{\int_{0}^{\infty} \varepsilon_{\text {lower }}\left(n_{\nu, s p}-n_{\nu, b b}\right)(h \nu)^{2} d h \nu} \cong n_{r}^{2}\left(h \bar{\nu}_{s p}\right),
$$

which is approximately equal to the refractive index at the average energy of the spontaneous emission spectrum above the effective bandgap, $E_{g}-E_{u}$, with

$$
\begin{aligned}
h \bar{\nu}_{s p} & =\frac{\int_{0}^{\infty} \alpha\left(n_{\nu, s p}-n_{\nu, b b}\right)(h \nu)^{3} d h \nu}{\int_{0}^{\infty} \alpha\left(n_{\nu, s p}-n_{\nu, b b}\right)(h \nu)^{2} d h \nu} \\
& \cong \frac{\int_{E_{g}-E_{u}}^{\infty}\left(n_{\nu, s p}-n_{\nu, b b}\right)(h \nu)^{3} d h \nu}{\int_{E_{g}-E_{u}}^{\infty}\left(n_{\nu, s p}-n_{\nu, b b}\right)(h \nu)^{2} d h \nu} \cong E_{g}-E_{u}+k T,
\end{aligned}
$$

where $\alpha$ is the absorption coefficient. Note that right hand side of Eq. (7) also gives the average energy separation of the photoexcited electron and hole populations.

As with effective absorptance, it is useful to move the emittance term out of the integral by defining two different energy independent effective emittances for the respective upper and lower surfaces as,

$$
\begin{aligned}
\bar{\varepsilon}_{\text {upper }} & \equiv \frac{\int_{0}^{\infty} \varepsilon_{\text {upper }}\left(n_{\nu, s p}-n_{\nu, b b}\right)(h \nu)^{2} d h \nu}{\int_{E_{g}-E_{u}}^{\infty}\left(n_{\nu, s p}-n_{\nu, b b}\right)(h \nu)^{2} d h \nu} \\
& \cong \frac{\int_{0}^{\infty} \varepsilon_{\text {upper }} e^{-h \nu / k T}(h \nu)^{2} d h \nu}{k T\left(E_{g}-E_{u}+k T\right)^{2} e^{-\left(E_{g}-E_{u}\right) / k T}}, \\
\bar{\varepsilon}_{\text {lower }} & \equiv \frac{\int_{0}^{\infty} \varepsilon_{\text {lower }}\left(n_{\nu, s p}-n_{\nu, b b}\right)(h \nu)^{2} d h \nu}{\int_{E_{g}-E_{u}}^{\infty}\left(n_{\nu, s p}-n_{\nu, b b}\right)(h \nu)^{2} d h \nu} \\
& \cong \frac{\int_{0}^{\infty} \varepsilon_{\text {lower }} e^{-h \nu / k T}(h \nu)^{2} d h \nu}{k T\left(E_{g}-E_{u}+k T\right)^{2} e^{-\left(E_{g}-E_{u}\right) / k T}}
\end{aligned}
$$

Note that in a manner similar to absorptance, detailed balance models typically utilize a step-function emittance that is zero below the bandgap and unity above the bandgap, which presupposes that the absorptance and emittance are the same. However, for a typical solar cell they are not, because the energy range of incident solar radiation is much broader than the emission spectrum that typically occurs over energies where the absorption coefficient is small.

Using the Boltzmann approximation for low injection (i.e., $E_{g}-q V>3 k T$ ), the photon occupation number is given by a simple exponential, which is used to obtain the right-hand terms in Eqs. (5), (7), and (8).

It is convenient to define the radiative recombination saturation-current density as,

$$
J_{B} \equiv \frac{q 2 \pi k T}{h^{3} c^{2}}\left(\bar{\varepsilon}_{\text {upper }}+\bar{\varepsilon}_{\text {lower }} n_{g}^{2}\right)\left(E_{g}-E_{u}+k T\right)^{2},
$$

with the radiative recombination current written as,

$$
J_{\text {rad }} \cong J_{B} \cdot e^{-\left(E_{g}-E_{u}\right) / k T}\left(e^{q V / k T}-1\right) .
$$

Here, the radiative recombination saturation-current density is not simply dependent on bandgap energy as in the detailed balance model, however, it is also dependent on the material properties and structure geometry, including the refractive index, tail width, and junction thickness.

The preceding equations provide an important insight into the impact of tail states on solar cells: For a given voltage, the presence of tail states substantially increases the radiative recombination current by roughly a factor, $e^{E_{u} / k T}$, which is 1.29 and 1.37 for crystalline GaAs and $\mathrm{Si}$, respectively, using published values of the Urbach parameter, $E_{u}{ }^{22,23}$ The enhancement of spontaneous emission by states 
below the bandgap effectively shifts the optical absorption/ emission cutoff to lower energies, and the operating and open-circuit voltages decrease in a response to limit the spontaneous emission losses. For a given radiative recombination current, the voltage output is reduced by the width of the tail as quantified by comparing the exponents in Eq. (10).

The question as to whether states below the band edge improve solar cell performance depends on the trade-off between a higher short-circuit current and lower open-circuit voltage. For single-junction solar cells with a bandgap greater than optimal $\left(E_{g}>1.37 \mathrm{eV}\right)$, there is an increase in the energy conversion efficiency when absorption below the bandgap is present. On the contrary, when the bandgap is optimal or less than optimal the efficiency decreases when states below the band edge are present. Consequently, the existence or deliberate insertion of states below the band edge provides a method to realize an optimal absorption cutoff in the case when an ideal bandgap material is not available; however, they do not outperform an optimal bandgap material without the presence of these states. In addition, the preceding discussion ignores any increases in non-radiative recombination for a material with a large number of tail states.

It is interesting to point out that the radiative recombination saturation-current can also be written in terms of the radiative recombination coefficient, $B$, that is often used to describe the performance of many other optoelectronic devices, ${ }^{6}$ with $J_{B} \equiv q d B \gamma_{e}\left(N_{c} N_{v}\right)$, which is written in the same form as in the nonradiative recombination terms described in Eqs. (19) and (20), with the junction thickness, $d$, explicitly shown. Upon substituting the analytical expression for $B,{ }^{24}$ and using $\gamma_{e, \text { upper }}=\bar{\varepsilon}_{\text {upper }} /\left(4 \alpha_{g} d \cdot n_{g}^{2}\right)$ and $\gamma_{e, \text { lower }}$ $=\bar{\varepsilon}_{\text {lower }} /\left(4 \alpha_{g} d\right)$, Eq. (9) is obtained, where $\alpha_{g}$ is the absorption coefficient at the average photon energy of the spontaneous emission spectrum. In Eq. (9) the junction thickness is implicit in the emittance term and disappears in the optically-thick limit due to photon recycling.

\section{Four planar structures}

This work categorizes solar cell structures into four different planar configurations. Schematic diagrams of these structures are shown in Fig. 3, where Structure A (Fig. 3(a)) is a semiconductor slab with a non-reflecting index-matched absorbing substrate, Structure B (Fig. 3(b)) is a semiconductor slab with an ideal $100 \%$ reflecting substrate, Structure C (Fig. 3(c)) is a textured semiconductor slab with a nonreflecting index-matched absorbing substrate, and Structure D (Fig. 3(d)) is a textured semiconductor slab with an ideal $100 \%$ reflecting substrate. Here, the term "reflecting substrate" is used to describe a reflecting layer on the backside of the device, which can be, for example, a highly-reflective metal layer. The terms "upper surface" and "lower surface" in this study are used to describe the surface facing the sun light and the bottom interface between the slab and the substrate, respectively. Moreover, it is assumed that there is no reflection at the upper surface (i.e., $T_{\alpha}=1-A_{\alpha}$ ) since the reflection can be substantially reduced using an antireflection coating and/or surface roughening. (a)

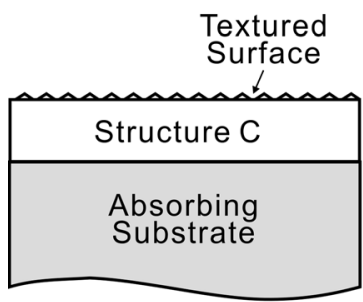

(c)

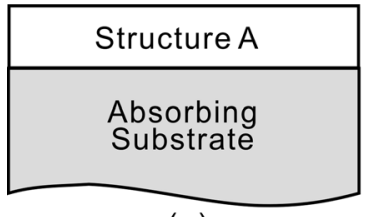

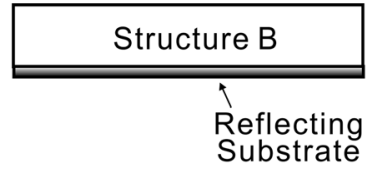

(b)

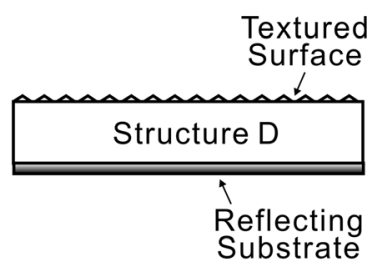

(d)
FIG. 3. Schematic diagrams of four types of planar solar cell structures, labeled Structures A, B, C, and D. Structure A is a semiconductor slab with an absorbing substrate, Structure B is a semiconductor slab with a reflecting substrate, Structure C is a textured semiconductor slab with an absorbing substrate, and Structure D is a textured semiconductor slab with a reflecting substrate.

Structure A has a smooth upper surface and an absorbing substrate which typifies a junction (i.e., a subcell) inside a multi-junction solar cell where the adjacent lower junction functions as an absorbing substrate. Structure D has a textured upper surface and a reflecting substrate which typifies a high performance single-junction solar cell, especially for a Si single-junction cell. ${ }^{25}$ Structure B has a smooth top surface and a reflecting substrate and Structure $C$ has a textured top surface and an absorbing substrate, which are investigated for comparison.

The absorptance of a given junction is a function of the absorption coefficient, junction thickness, and surface/interface properties. Using ray optics, the absorptance is given by,

$$
\begin{aligned}
A_{\alpha} \cong 1-e^{-\alpha \cdot d} & \text { for Structure A, } \\
A_{\alpha} \cong 1-e^{-\alpha \cdot 2 d} & \text { for Structure B, } \\
A_{\alpha}=1-t_{\alpha} & \text { for Structure C } \\
A_{\alpha}=\frac{1-t_{\alpha}}{1-\left(1-\frac{1}{n_{r}^{2}}\right) t_{\alpha}} & \text { for Structure D, }
\end{aligned}
$$

where $\alpha$ is the absorption coefficient, $d$ is the slab thickness, $n_{r}$ is the refractive index of the semiconductor slab, and $t_{\alpha}$ is the fraction of incident photons not absorbed on a single pass through the slab, with

$$
\begin{aligned}
t_{\alpha} & =\int_{0}^{\pi / 2} e^{-\alpha d / \cos \theta} 2 \cos \theta \sin \theta d \theta \text { for Structure C, } \\
t_{\alpha} & =\int_{0}^{\pi / 2} e^{-2 \alpha d / \cos \theta} 2 \cos \theta \sin \theta d \theta \text { for Structure } \mathrm{D}
\end{aligned}
$$

where $\theta$ is the angle between the scattered ray and the surface normal. Maximal (i.e., Lambertian) scattering is assumed, where $2 \cos \theta$ is the probability that a ray is scattered into the solid angle, $\sin \theta d \theta$. Here, $2 \cos \theta$ can be 
replaced by other scattering functions for non-Lambertian scattering. For Structures A and B, the optical path length is slightly longer when the incident angle of the solar radiation is not normal, however, the difference is negligible for typical semiconductors due to their large indices of refraction.

Since much of the scattered light is trapped in Structure $\mathrm{D}$, the absorptance is greatly enhanced through the multiple reflection/scattering events. For randomized scattering from a textured top surface, Tiedje et ll $^{4}$ provide an analysis for the absorptance where the optical thickness of semitransparent layers is increased by a factor of $4 n_{r}^{2}$ on average, and their equation for absorptance in Structure $\mathrm{D}$ is $\left[1+1 /\left(4 n_{r}^{2} \alpha d\right)\right]^{-1}$, which is a good approximation to Eq. (11d) with a difference of less than $2 \%$ when the refractive index is greater than 3 .

Using crystalline $\mathrm{Si}$ and $\mathrm{GaAs}$ as the prototypical indirect and direct bandgap semiconductors, the effective absorptance given by Eq. (3) as a function of slab thickness is plotted in Fig. 4 for the four slab structures. Here the AM1.5 $\mathrm{G}$ solar spectrum is used in the calculation; there is only a slight difference in the results if the AM0 spectrum ${ }^{26}$ is used. Published values for the refractive index, $n_{r}$, absorption coefficient, $\alpha{ }^{24}$ and the tail widths, $E_{u}=8.5 \mathrm{meV}$ (Ref. 22) and $6.7 \mathrm{meV}$ (Ref. 23) are used for $\mathrm{Si}$ and GaAs, respectively. The slab thicknesses for $\overline{A_{\alpha}}=0.90,0.99$, and 1.0 are also summarized in Table I, where, for a given $\overline{A_{\alpha}}$, Structure A is the thickest, Structure C is the second thickest, Structure B is the third thickest, and Structure D is the thinnest. Moreover, direct bandgap junctions are much thinner than indirect bandgap junctions.

Most reported GaAs single-junction solar cells that utilize the Structure A configuration ${ }^{28}$ satisfy $\overline{A_{\alpha}} \approx 1$. On the contrary, most reported high-performance Si single-junction solar cells ${ }^{25}$ that utilize the Structure D configuration to further increase the optical-thickness of the junction also satisfy $\overline{A_{\alpha}} \approx 1$. From a practical device design point of view, further increases of the effective absorptance beyond $\overline{A_{\alpha}} \approx 1$ typically result in excessive non-radiative recombination and parasitic absorption losses. Note that the absorptance calculation assumes relatively low injection levels (i.e., $E_{g}-q V>3 k T$ ), as the absorption coefficient at equilibrium is used. Low injection is a valid assumption for most solar cells and is used throughout this study.
TABLE I. Slab thicknesses for the effective absorptance, $\overline{A_{\alpha}}=0.90,0.99$, and 1.00 , for $\mathrm{Si}$ and GaAs.

\begin{tabular}{|c|c|c|c|c|}
\hline$\overline{A_{\alpha}}$ & Structure A & Structure B & Structure C & Structure D \\
\hline \multicolumn{5}{|c|}{ Slab thickness for $\mathrm{Si}(\mathrm{mm})$} \\
\hline 0.90 & 0.26 & 0.13 & 0.16 & 0.01 \\
\hline 0.99 & 2.95 & 1.48 & 1.94 & 0.16 \\
\hline 1.00 & 5.60 & 2.80 & 3.76 & 0.36 \\
\hline \multicolumn{5}{|c|}{ Slab thickness for GaAs $(\mu \mathrm{m})$} \\
\hline 0.90 & 0.99 & 0.50 & 0.66 & 0.05 \\
\hline 0.99 & 2.59 & 1.30 & 1.81 & 0.20 \\
\hline 1.00 & 3.44 & 1.72 & 2.40 & 0.28 \\
\hline
\end{tabular}

Using ray optics, ${ }^{21}$ it can be derived that the upper surface emittance for the four structures is,

$$
\varepsilon_{\text {upper }}=1-n_{r}^{2} \int_{0}^{\theta_{c}} e^{-\alpha d / \cos \theta} 2 \cos \theta \sin \theta d \theta \quad \text { for Structure A, }
$$

$$
\varepsilon_{\text {upper }}=1-n_{r}^{2} \int_{0}^{\theta_{c}} e^{-2 \alpha d / \cos \theta} 2 \cos \theta \sin \theta d \theta \quad \text { for Structure } \mathrm{B}
$$

$$
\varepsilon_{\text {upper }}=A_{\alpha} \quad \text { for Structures } \mathrm{C} \text { and D, }
$$

where in Structures A and B, $\theta$ is the angle between the surface normal and the emitted ray inside the junction and $\theta_{c}=\sin ^{-1}\left(1 / n_{r}\right)$ is the critical angle beyond which the emitted rays do not escape from the upper surface. Since the angular integration for Structures A and B is done over the escape cone inside the slab, the refractive index squared appears in front of the integral. If the integration is done outside the slab overall angles, the refractive index does not appear, however, the expression for the path length in terms of the outside angle is more complicated due to refraction at the surface. For Structures C and D, the emittance equals the absorptance given in Eq. (13c), since the maximal scattering path length is the same during either absorption or emission in textured structures.

On the contrary, for Structures A and B, the emittance is slightly larger than the absorptance given in Eqs. (13a) and (13b), because the absorption path length under normal incidence solar radiation is slightly shorter when compared to
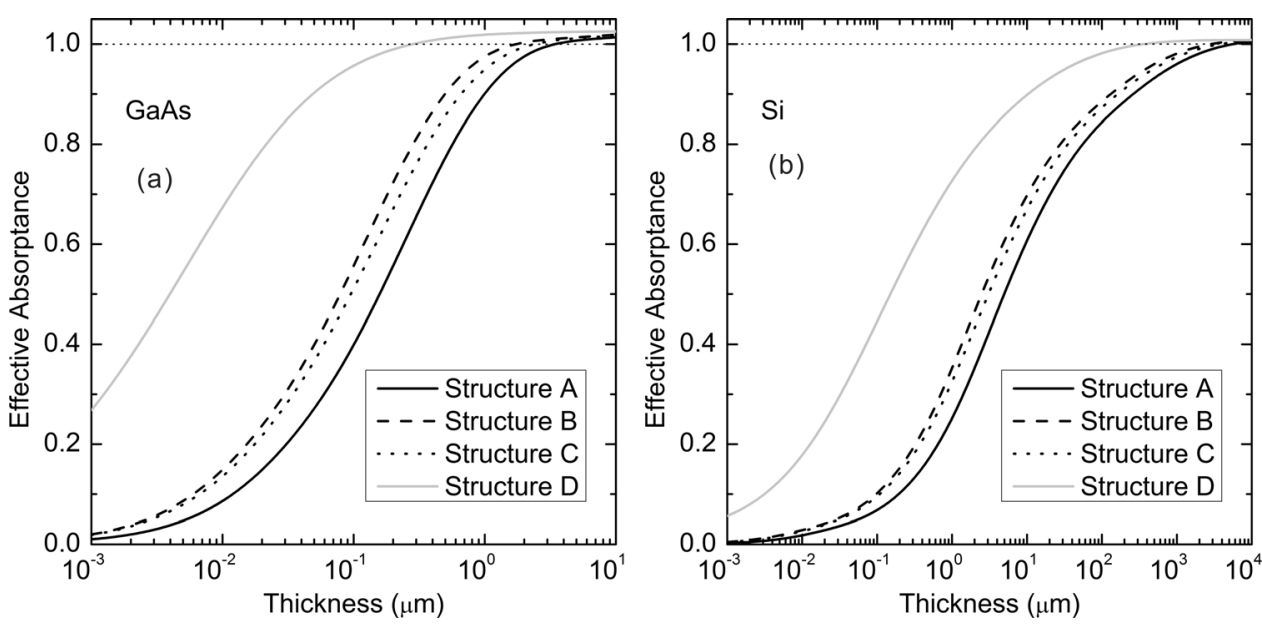

FIG. 4. Effective absorptance, $\overline{A_{\alpha}}$, vs physical thickness, $d$, for the four types of semiconductor slabs investigated: (a) $\mathrm{GaAs}$, and (b) Si. 

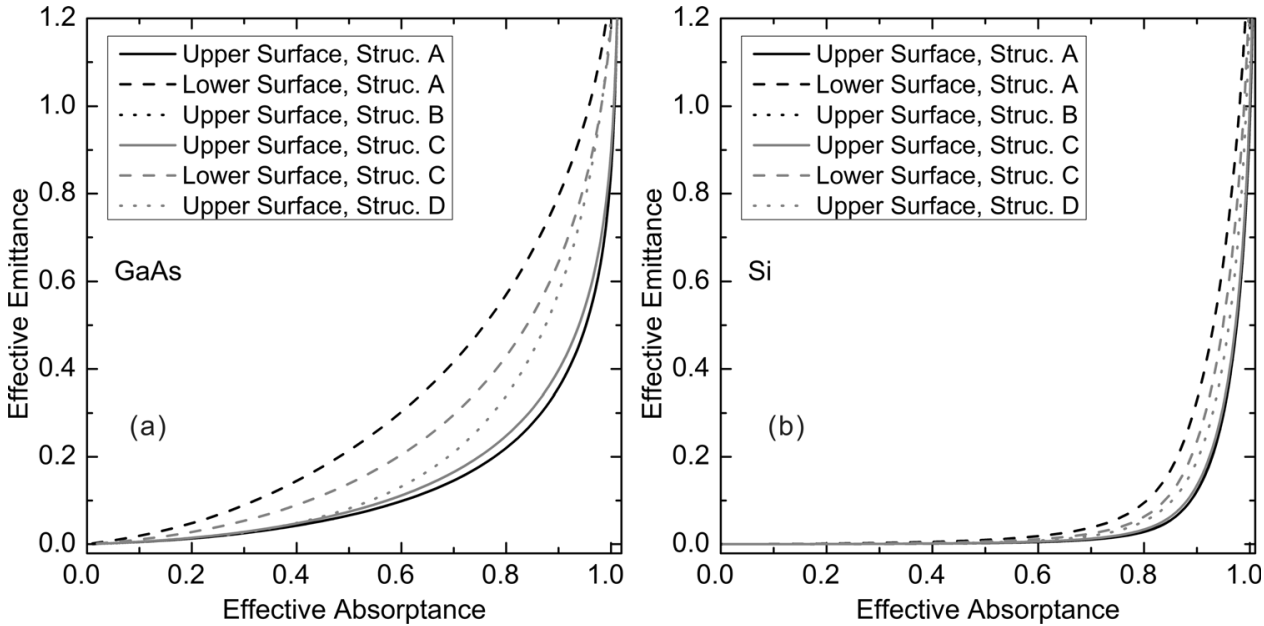

FIG. 5. Effective emittance, $\bar{\varepsilon}_{\text {upper }}$ and $\bar{\varepsilon}_{\text {lower }}$, vs physical thickness, $d$, for the four types of semiconductor slabs investigated: (a) GaAs, and (b) Si. the emission which occurs at all angles within the escape cone. Under maximum concentration ( $\sim 7000$ suns) where the solar radiation is incident at all angles, the absorptance is identical to the emittance as the solar radiation is absorbed at all angles within the escape cone.

In Structures B and D, the reflecting substrate results in zero emission escaping from the lower surface. However, in Structures A and C a large amount of spontaneous emission is coupled into the absorbing substrate as it is index matched. Furthermore, the upper surface in both structures reflects $1-1 / n_{r}^{2}$ of the internal spontaneous emission incident on it, which is substantial when the refractive index is large. The flux is $n_{r}^{2}$ times larger inside the semiconductor compared to free space, with

$$
\begin{gathered}
\varepsilon_{\text {lower }}=1-\int_{0}^{\theta_{c}} e^{-\alpha d / \cos \theta} 2 \cos \theta \sin \theta d \theta \\
-\int_{\theta_{c}}^{\pi / 2} e^{-2 \alpha d / \cos \theta} 2 \cos \theta \sin \theta d \theta \quad \text { for Structure A, } \\
\varepsilon_{\text {lower }}=0 \quad \text { for Structure B } \\
\varepsilon_{\text {lower }}=\left(1-t_{\alpha}\right)\left[1+t_{\alpha}\left(1-\frac{1}{n_{r}^{2}}\right)\right] \text { for Structure C } \\
\varepsilon_{\text {lower }}=0 \text { for Structure D. }
\end{gathered}
$$

\begin{tabular}{|c|c|c|c|c|c|c|c|c|}
\hline \multirow[b]{2}{*}{$\overline{A_{\alpha}}$} & \multicolumn{2}{|c|}{ Structure A } & \multicolumn{2}{|c|}{ Structure B } & \multicolumn{2}{|c|}{ Structure C } & \multicolumn{2}{|c|}{ Structure D } \\
\hline & $\bar{\varepsilon}_{\text {upper }}$ & $\bar{\varepsilon}_{\text {lower }}$ & $\bar{\varepsilon}_{\text {upper }}$ & $\bar{\varepsilon}_{\text {lower }}$ & $\bar{\varepsilon}_{\text {upper }}$ & $\bar{\varepsilon}_{\text {lower }}$ & $\bar{\varepsilon}_{\text {upper }}$ & $\bar{\varepsilon}_{\text {lower }}$ \\
\hline \multicolumn{9}{|c|}{ Effective emittance for $\mathrm{Si}$} \\
\hline 0.90 & 0.12 & 0.33 & 0.12 & 0 & 0.13 & 0.24 & 0.19 & 0 \\
\hline 0.99 & 0.72 & 1.17 & 0.72 & 0 & 0.75 & 1.02 & 0.92 & 0 \\
\hline 1.00 & 0.97 & 1.44 & 0.97 & 0 & 1.01 & 1.29 & 1.24 & 0 \\
\hline \multicolumn{9}{|c|}{ Effective emittance for GaAs } \\
\hline 0.90 & 0.36 & 0.79 & 0.36 & 0 & 0.40 & 0.64 & 0.58 & 0 \\
\hline 0.99 & 0.72 & 1.18 & 0.72 & 0 & 0.77 & 1.06 & 1.08 & 0 \\
\hline 1.00 & 0.85 & 1.30 & 0.85 & 0 & 0.90 & 1.18 & 1.20 & 0 \\
\hline
\end{tabular}

TABLE II. Effective emittance when effective absorptance is $\overline{A_{\alpha}}=0.90$, 0.99 , and 1.00 , for Si and GaAs.
The effective emittance versus effective absorptance for GaAs and Si is shown in Figs. 5(a) and 5(b). For the same effective absorptance, the effective emittance of the lower surface for Structure A is the largest, the lower surfaces for Structure C are the second largest, the upper surface of Structure D is the third largest, the upper surface of Structure $\mathrm{C}$ is the fourth largest, and the upper surfaces for Structures $\mathrm{A}$ and $\mathrm{B}$ is the smallest (they almost overlap in the plot). Moreover, in order to achieve the same effective emittance, indirect bandgap junctions are much thicker than direct bandgap junctions. In addition, it is interesting to point out that the preceding calculations are useful for other optoelectronic devices such as light emitting diodes, for which Structure D is preferred because it has a much higher emittance than the other three structures for the same slab thickness. The effective emittance for GaAs and $\mathrm{Si}$ are also summarized in Table II for the cases where the effective absorptance is $\overline{A_{\alpha}}=0.90,0.99$, and 1.00 .

Figure 6 shows the average refractive index, $n_{g}$, versus bandgap energy for some common group-IV, III-V, and

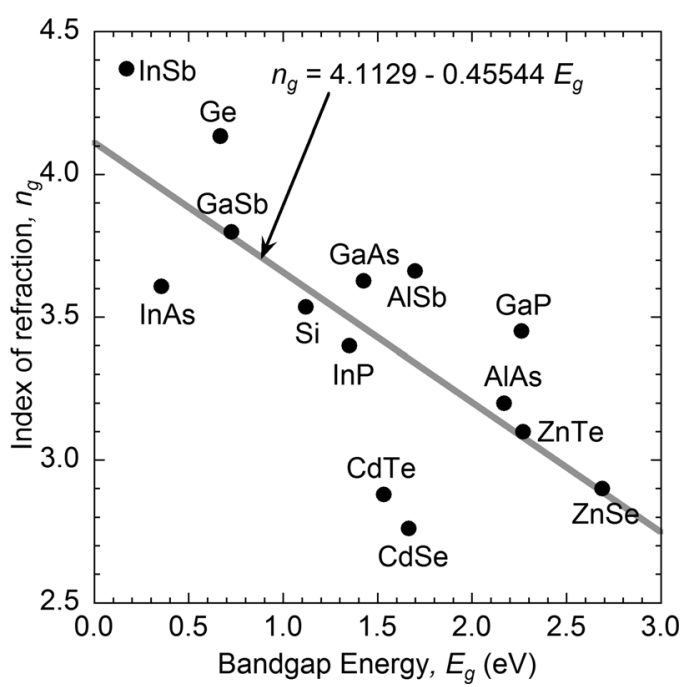

FIG. 6. Refractive index at the average spontaneous emission energy, $E_{g}+k T$, vs bandgap energy, $E_{g}$, for the common II-VI, III-V, and group IV semiconductors shown. The solid line is a least squares fit to the data that provides an average refractive index as a function of the bandgap energy for common semiconductor materials. 


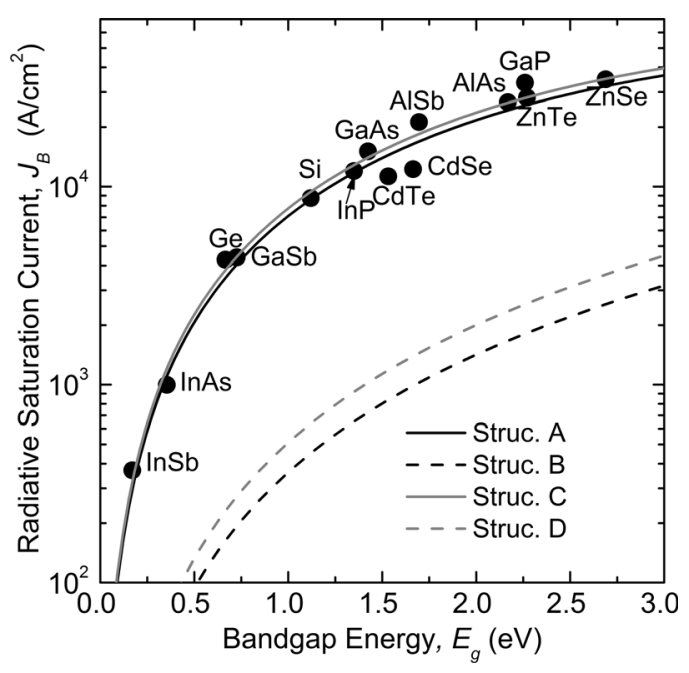

FIG. 7. Radiative recombination saturation current density, $J_{B}$, vs bandgap energy, $E_{g}$, for solar cells with absorbing substrates (Structure A) and textured surfaces and reflecting substrates (Structure D). The solid circles are calculations for Structure A using the discrete values of the refractive index for the common II-VI, III-V, and group IV semiconductors. The solid curves (Structures $\mathrm{A}$ and $\mathrm{C}$ ) are calculated using the average refractive index as a function of the bandgap energy and the dashed curves (Structures B and D) do not depend on the refractive index.

II-VI semiconductors based on published data. ${ }^{27}$ The solid gray line is a linear fit to $n_{g}$ with $n_{g}=4.12-0.46 \cdot E_{g}$ (where $E_{g}$ is in units of $\mathrm{eV}$ ).

The radiative recombination saturation current density as a function of bandgap energy for the four structures is evaluated and plotted in Fig. 7, using the effective emittances for GaAs given by $\overline{A_{\alpha}}=1$ (see Table II). The solid circles are the calculations for Structure A using published values of $n_{g} .{ }^{27}$ The solid curves for Structures $\mathrm{A}$ and $\mathrm{C}$ give the results using the linear fit for $n_{g}$; here, the curves nearly coincide because the radiative loss for both is dominated by the absorbing substrate (see Eq. (9)). On the contrary, the curves for Structures B and D with the reflecting substrates are much lower and do not depend on the refractive index since the emittance of the lower surface is zero. As can be seen from Fig. 7, the radiative recombination saturationcurrent density, $J_{B}$, increases with the bandgap energy, $E_{g}$, and is substantially larger for Structures A and C as spontaneous emission is coupled into the substrate.

\section{E. Non-radiative recombination current densities}

To further evaluate non-radiative recombination losses in solar cells, it is necessary to establish simple models for the SRH and Auger recombination currents in solar cells. The SRH and Auger recombination current densities are given by,

$$
\begin{gathered}
J_{S R H}=q d R_{S R H}, \\
J_{\text {Auger }}=q d R_{\text {Auger }},
\end{gathered}
$$

where $R_{S R H}$ and $R_{\text {Auger }}$ are SRH and Auger recombination rates, ${ }^{6}$ respectively.

Under low injection, Eqs. (15) and (16) are approximated by,

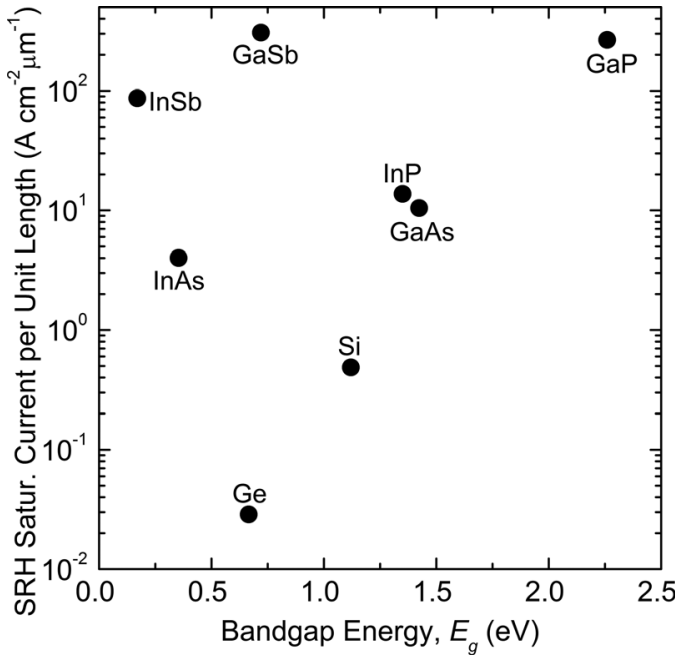

FIG. 8. Lowest reported SRH recombination saturation current density per unit length, $J_{A} / d$, vs bandgap energy, $E_{g}$, for the various semiconductors shown.

$$
\begin{aligned}
& J_{S R H} \approx J_{A} e^{-\left(E_{g}-E_{u}\right) / 2 k T} e^{-q V / 2 k T}\left(e^{q V / k T}-1\right), \\
& J_{\text {Auger }} \approx J_{C} e^{-3\left(E_{g}-E_{u}\right) / 2 k T} e^{q V / 2 k T}\left(e^{q V / k T}-1\right),
\end{aligned}
$$

where $J_{A}$ and $J_{C}$ are $\mathrm{SRH}$ and Auger recombination saturation current densities, and $E_{g}-E_{u}$ is the effective bandgap described in Eq. (10). In the limiting case when the semiconductor material in question is intrinsic, $J_{A}$ and $J_{C}$ are given by,

$$
\begin{gathered}
J_{A}=q d A \sqrt{N_{c} N_{v}}, \\
J_{C}=q d C\left(N_{c} N_{v}\right)^{3 / 2},
\end{gathered}
$$

where $A$ is the SRH recombination coefficient, $C$ is the Auger recombination coefficient, and $N_{c}$ and $N_{v}$ are the conduction- and valence-band effective density of states, respectively. ${ }^{6}$

It is informative to look at the extent of the SRH and Auger recombination for some common group IV and III-V semiconductors. Following Eq. (19), the SRH recombination saturation current density per unit length versus bandgap energy is calculated and shown in Fig. 8, where the published longest minority carrier lifetime for each material is used. ${ }^{29}$ The results vary from 0.1 to $300 \mathrm{~A} \mathrm{~cm}^{-2} \mu \mathrm{m}^{-1}$ with no comprehensive trend. Similarly, following Eq. (20), the Auger recombination saturation current density per unit length versus bandgap energy is shown in Fig. 9, where published values of the Auger recombination coefficients are used. $^{29}$ The results vary from 100 to $300000 \mathrm{~A} \mathrm{~cm}^{-2} \mu \mathrm{m}^{-1}$; $\mathrm{Si}$ has the largest value, which is mainly due to its large effective densities of states.

\section{F. Loss and extracted power}

The equations for single-junction solar cells including extracted power, conversion efficiency, and losses are listed in Table III. Where the extractable energy of the photogenerated carriers is $q V$, the average energy separation of the 


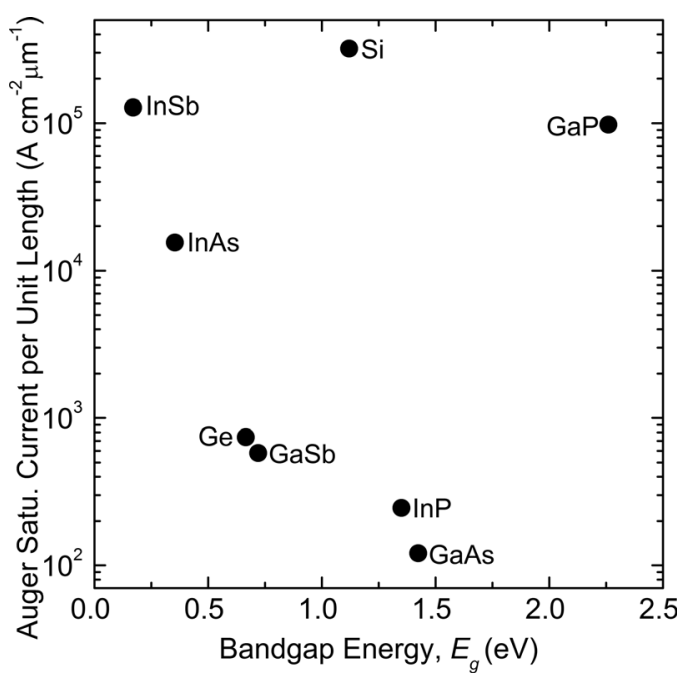

FIG. 9. Lowest reported Auger recombination current density per unit length, $J_{C} / d$, vs bandgap energy, $E_{g}$, for the various semiconductors shown.

electron and hole populations is $E_{g}-E_{u}+k T$, and the average energy of the absorbed solar radiation is,

$$
\overline{h \nu}_{\text {sun }}=\frac{\int_{0}^{\infty} A_{\alpha} n_{\text {sun }} h \nu d h \nu}{\int_{0}^{\infty} A_{\alpha} n_{\text {sun }} d h \nu},
$$

which monotonically deceases as the junction thickness increases, saturating at $\overline{A_{\alpha}} \approx 1$. The Boltzmann approximation provides $E_{g}-E_{u}+k T$ as the average energy separation of the electron and hole populations and consequently, the average energy of the internal spontaneous emission (see Eq. (7)). Note that the emitted spontaneous emission is spectrally red-shifted as it undergoes photon recycling and, in general, has an average energy less than $E_{g}-E_{u}+k T$. In this study, precise numerical calculations are used to calculate the average emitted photon energy for GaAs and Si single junction cases.

The transmission and thermalization losses are independent of the voltage, while the spatial-relaxation and recombination losses are dependent upon the voltage. The trade-off between spatial-relaxation and recombination loss determines the optimal operating condition (maximum power output) for solar cells. The spatial-relaxation loss is maximum (minimum) and the recombination loss is minimum (maximum) at the short-circuit (open-circuit) condition.

\section{G. Application to multi-junction solar cells}

The preceding model is further extended to multijunction solar cells consisting of $j(j \geq 1)$ junctions numbered in ascending order from top to bottom (i.e., from the largest bandgap to the smallest bandgap). The $i$ th junction has thickness, $d$, bandgap energy, $E_{g}$, Urbach tail width, $E_{u}$, absorptance, $A_{\alpha}$, and emittance, $\varepsilon_{\text {upper }}$ and $\varepsilon_{\text {lower }}$. In the following, the superscript, $i$, is used as necessary for clarity and left out where the context makes it apparent. The current density for the $i$ th junction is,

$$
J^{i}=J_{s c}^{i}-\left(J_{\text {rad }}^{i}+J_{S R H}^{i}+J_{\text {Auger }}^{i}\right) \text { for } 1 \leq i \leq j
$$

which shares the same form as that of single-junction solar cells. The radiative, SRH, and Auger recombination current

\begin{tabular}{|c|c|}
\hline Parameter & Definition and equation \\
\hline \multirow[t]{2}{*}{ Extracted power } & Power extracted at maximum output condition \\
\hline & $p_{\text {out }}=J_{m} V_{m}$ \\
\hline \multirow{4}{*}{ Conversion efficiency } & Extracted power \\
\hline & $\overline{\text { Incident solar power }}$ \\
\hline & $\eta=\frac{P_{\text {out }}}{=}=\frac{J_{m} V_{m}}{}$ \\
\hline & $\eta=\overline{P_{\text {in }}}=\overline{\int_{0}^{\infty} n_{\text {sun }} \cdot h v \cdot d h v}$ \\
\hline \multirow[t]{2}{*}{ Transmission loss } & Solar power not absorbed by solar cell \\
\hline & $L_{t r}=\int_{0}^{\infty}\left(1-A_{\alpha}\right) \cdot n_{\text {sun }} \cdot h v \cdot d h v=P_{\text {in }}-\frac{J_{s c}}{h} \cdot \overline{h v}_{\text {sun }}$ \\
\hline \multirow[t]{2}{*}{ Thermalization loss } & Energy lost as photoexcited carriers equilibrate to their respective populations \\
\hline & $L_{t h}=\int_{0}^{\infty} A_{\alpha} n_{s u n} \cdot\left[h v-\left(E_{g}-E_{u}+k T\right)\right] d h v=\frac{J_{s c}}{a} \cdot\left[\overline{h v}_{s u n}-\left(E_{g}-E_{u}+k T\right)\right]$ \\
\hline \multirow[t]{2}{*}{ Spatial relaxation loss } & Potential energy lost as the electron-hole population is separated and swept to each respective contact \\
\hline & $L_{s r}=\frac{J}{q} \cdot\left[\left(E_{g}-E_{u}+k T\right)-q V\right]$ \\
\hline \multirow[t]{2}{*}{ Radiative loss } & Energy lost to radiative recombination \\
\hline & $L_{r a d}=\frac{J_{r a d}}{q} \cdot\left(E_{g}-E_{u}+k T\right)$ \\
\hline \multirow[t]{2}{*}{ SRH loss } & Energy lost to SRH recombination \\
\hline & $L_{S R H}=\frac{J_{S R H}}{} \cdot\left(E_{g}-E_{u}+k T\right)$ \\
\hline \multirow{3}{*}{ Auger loss } & Energy lost to Auger recombination \\
\hline & $L_{\text {Auger }}=\underline{J_{\text {Auger }}} \cdot\left(E_{g}-E_{u}+k T\right)$ \\
\hline & $L_{\text {Auger }}-\frac{-}{q} \cdot\left(L_{g}-L_{u}+\kappa I\right)$ \\
\hline
\end{tabular}

TABLE III. Definitions and equations for losses and extracted power in single-junction solar cells. 
densities for the $i$ th junction are also the same as these for single-junctions solar cells (see Eqs. (10), (17), and (18)).

The short-circuit current density becomes complicated when more than one junction is present. Carriers are generated by the absorption of both solar radiation, $n_{\text {sun }}^{i}$, and coupled spontaneous emission from the adjacent largerbandgap $i-1$ junction, ${ }^{9-12} n_{s p \text {,lower }}^{i-1}$, where $N_{s p, l o w e r}^{i-1}$ $=\int_{0}^{\infty} n_{s p, l o w e r}^{i-1} d h \nu$ (with $\left.n_{s p, l o w e r}^{0}=0\right)$ is the total spontaneous emission received, which is typically completely absorbed, since it has energies well above the bandgap of the $i$ th junction where the absorption coefficient is sufficiently large. Taking into consideration the coupled spontaneous emission from the lower surface of the preceding larger-bandgap junction, the short-circuit current density per unit area of the $i$ th junction is,

$$
\begin{aligned}
J_{s c}^{i} & =q \int_{0}^{\infty} A_{\alpha}\left(n_{s u n}^{i}+n_{s p, l o w e r}^{i-1}\right) d h \nu \\
& \cong q\left(\overline{A_{\alpha}} \int_{E_{g}-E_{u}}^{\infty} n_{s u n}^{i} d h \nu+N_{s p, l o w e r}^{i-1}\right) \text { for } 1 \leq i \leq j
\end{aligned}
$$

where $n_{\text {sun }}^{i}=T_{\alpha}^{i-1} n_{\text {sun }}^{i-1}$ for $2 \leq i \leq j$ is the solar radiation incident on the $i$ th junction and $T_{\alpha}^{i}$ is the transmittance of $i$ th junction, with $T_{\alpha}^{0}=1$ and $n_{\text {sun }}^{1}=n_{\text {sun }}$ as the solar radiation incident on the top junction. When $\overline{A_{\alpha}}=1$ is satisfied, each junction is equivalent to a long-pass "filter" with a cut-off at $E_{g}^{i}-E_{u}^{i}$ for the solar radiation spectrum which leads to $J_{s c}^{i}=q\left(\int_{E_{g}^{i}-E_{u}^{i}}^{E_{u}^{i-1}-E^{i-1}} n_{s u n}+N_{s p, l o w e r}^{i-1}\right) \quad\left(\right.$ with $\left.\quad E_{g}^{0}-E_{u}^{0}=\infty\right)$, assuming the bandgap separation between the junctions are much greater than the absorption tail width. Note that the condition, $\overline{A_{\alpha}}=1$, provides an opportunity to generally investigate multi-junction solar cells without knowing the absorption coefficient and thickness for each junction.

Moreover, to study the best performance for devices, it is assumed that the multi-junction solar cell in question is current-matched at the maximum power output condition for each junction. The total power extracted is determined by summing overall junctions, with

$$
P_{\text {out }}=J_{m} \sum_{i=1}^{j} V_{m}^{i} .
$$

Additionally, the energy conversion efficiency is,

$$
\eta=\frac{P_{\text {out }}}{P_{\text {in }}}=\frac{J_{m} \sum_{i=1}^{j} V_{m}^{i}}{\int_{0}^{\infty} n_{\text {sun }} h \nu \times d h \nu} .
$$

The total SRH, Auger, and spatial-relaxation losses are also obtained by summing each of them overall junctions, with

$$
\begin{gathered}
L_{S R H}=\sum_{i=1}^{j} \frac{J_{S R H}^{i}}{q}\left(E_{g}^{i}-E_{u}^{i}+k T\right), \\
L_{\text {Auger }}=\sum_{i=1}^{j} \frac{J_{\text {Auger }}^{i}}{q}\left(E_{g}^{i}-E_{u}^{i}+k T\right), \\
L_{s r}=\frac{J_{m}}{q} \sum_{i=1}^{j}\left[\left(E_{g}^{i}-E_{u}^{i}+k T\right)-q V_{m}^{i}\right] .
\end{gathered}
$$

The transmission loss is given by

$$
\begin{aligned}
L_{t r} & =\int_{0}^{\infty}\left(\prod_{1}^{j} T_{r}^{i}\right) n_{s u n} h \nu \times d h \nu \\
& \cong \int_{0}^{\infty} n_{\text {sun }}^{j} h \nu \times d h \nu-\overline{A_{\alpha}^{j}} \int_{E_{g}^{j}-E_{u}^{j}}^{\infty} n_{s u n}^{j} h \nu \times d h \nu
\end{aligned}
$$

which is approximately the transmission loss for the bottom junction, since typically the solar photons with energy above the bandgap of the bottom junction are completely absorbed. When $\overline{A_{\alpha}}=1$, the transmission loss is simplified to $L_{t r}=\int_{0}^{E_{g}^{j}-E_{u}^{j}} n_{\text {sun }}^{j} h \nu \times d h \nu$.

The thermalization loss includes the thermalization of both solar radiation and coupled spontaneous emission, and is written as,

$$
\begin{aligned}
L_{t h}= & \sum_{i=1}^{j}\left\{\int_{0}^{\infty} A_{\alpha}^{i}\left(n_{\text {sun }}^{i}+n_{s p, l o w e r}^{i-1}\right)\left[h \nu-\left(E_{g}^{i}-E_{u}^{i}+k T\right)\right] d h \nu\right\} \\
\cong & \sum_{i=1}^{j}\left\{\frac{J_{s c}^{i}}{q}\left[\overline{h \nu}_{\text {sun }}^{i}-\left(E_{g}^{i}-E_{u}^{i}+k T\right)\right]\right. \\
& \left.+N_{s p, l o w e r}^{i-1}\left[\left(E_{g}^{i-1}-E_{u}^{i-1}\right)-\left(E_{g}^{i}-E_{u}^{i}\right)\right]\right\}
\end{aligned}
$$

with $n_{s p \text {,lower }}^{0}=0$. When $\overline{A_{\alpha}}=1$, the average energy of the absorbed solar photons for the $i$ th junction is,

$$
\overline{h \nu}_{\text {sun }}^{i} \cong \int_{E_{g}^{i}-E_{u}^{i}}^{E_{g}^{i-1}-E_{u}^{i-1}} n_{\text {sun }} h \nu d h \nu / \int_{E_{g}^{i}-E_{u}^{i}}^{E_{g}^{i-1}-E_{u}^{i-1}} n_{\text {sun }} d h \nu,
$$

where $E_{g}^{0}-E_{u}^{0}=\infty$. Note that for a given bandgap, the average energy of the absorbed solar photons, and hence the thermalization losses, decrease as the junction number increases.

The total radiative loss is obtained by summing the spontaneous emission lost from the upper surfaces of all junctions and the lower surface of the bottom junction, and is written as,

$$
L_{s p}=\sum_{i=1}^{j-1} N_{s p, u p p e r}^{i}\left(E_{g}^{i}-E_{u}^{i}+k T\right)+\frac{J_{r a d}^{j}}{q}\left(E_{g}^{j}-E_{u}^{j}+k T\right) .
$$

Here, the spontaneous emission coupled into each lower junction is excluded as a loss (except for the bottom junction), since it is reasonably assumed to be completely absorbed in the adjacent smaller bandgap junctions.

\section{MODELING RESULTS AND DISCUSSION}

\section{A. Single-junction solar cells}

In this section, important design parameters such as junction thickness, solar concentration, bandgap, junction number, and material quality in terms of the SRH recombination current density per unit length are studied using the previously established equations.

Junction thickness is a critical design parameter for single-junction solar cells. To make the discussion more 

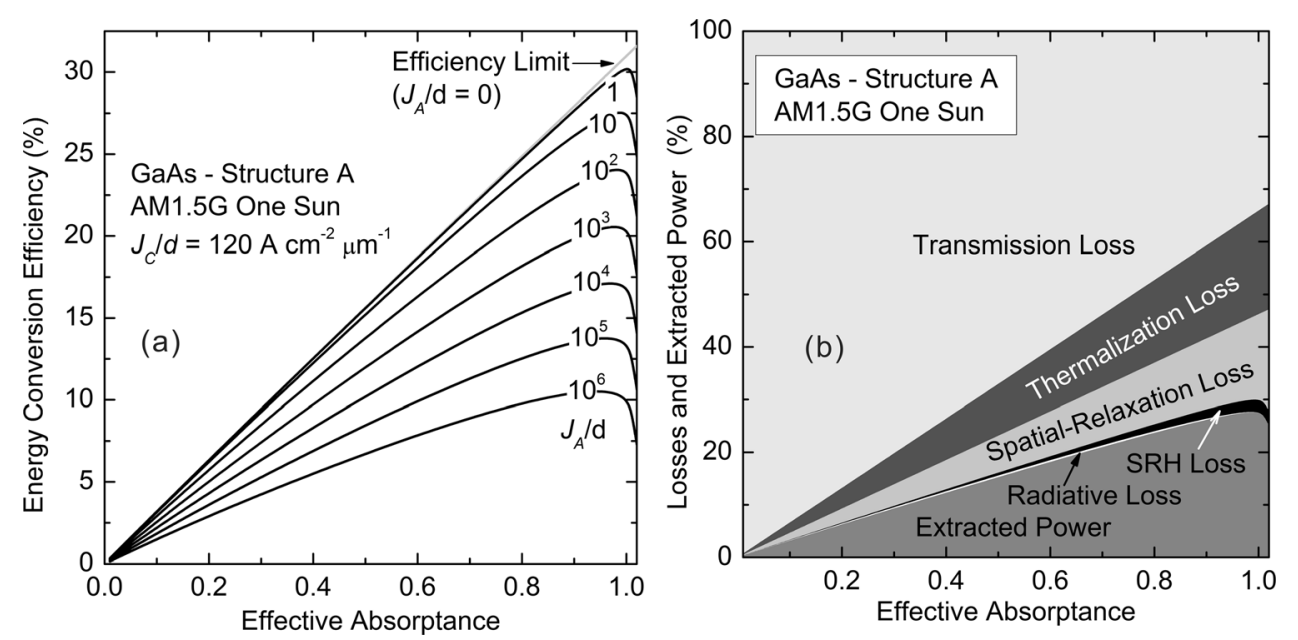

FIG. 10. Single-junction GaAs solar cell under one sun AM1.5 G with typical Auger recombination current density, $J_{C} / d=120 \mathrm{~A} \mathrm{~cm}^{-2} \mu \mathrm{m}^{-1}$. (a) Efficiency vs junction absorptance (i.e., optical thickness) for various SRH recombination current densities, $J_{A} / d$. (b) Solar power extracted and lost vs absorptance, assuming SRH recombination current density, $J_{A} / d=10 \mathrm{~A} \mathrm{~cm}^{-2} \mu \mathrm{m}^{-1}$. general and useful for practical devices, the effective absorptance (i.e., the "optical thickness" of a solar cell junction) is investigated, rather than the physical thickness of the junction, which is strongly material dependent. Using a GaAs single-junction solar cell with Structure A as an example, the energy conversion efficiency versus the effective absorptance for the AM1.5 G solar spectrum is plotted in Fig. 10(a) for various $\mathrm{SRH}$ recombination saturation current densities per unit length, $J_{A} / d$. In the calculation, a typical Auger recombination saturation current density per unit length of $J_{C} / d=120 \mathrm{~A} \mathrm{~cm}^{-2} \mu \mathrm{m}^{-1}$ is used, which is negligible compared to the other recombination losses. The gray curve with $J_{A}=0 \mathrm{~A} / \mathrm{cm}^{2}$ shows the efficiency limit with no $\mathrm{SRH}$ recombination. This efficiency limit monotonically increases as the short-circuit current monotonically increases with effective absorptance.

When SRH recombination is present, there is an optimal effective absorptance (i.e., junction thickness) given by a peak value in the efficiency. To further analyze the factors involved in this optimal junction thickness, the relative fractions of input power that are extracted and lost are shown by the areas under each curve in Fig. 10(b) versus the effective absorptance, where the best reported value of $J_{A} / d=10 \mathrm{~A} \mathrm{~cm}^{-2} \mu \mathrm{m}^{-1}$ for GaAs is used in the calculations. From Fig. 10(b) it can be seen that the transmission loss decreases linearly with the effective absorptance, while the SRH recombination and spatial-relaxation losses increase super-linearly with effective absorptance. The combination of these trends results in an optimal thickness for a singlejunction solar cell. Note that even for the best reported GaAs material quality, the Auger and radiative recombination losses are too small to be clearly seen in Fig. 10(b) and SRH recombination dominates the total recombination loss.

The optimal effective absorptance and energy conversion efficiency are plotted in Fig. 11(a) as a function of the SRH saturation current density per unit length, $J_{A} / d$, for the four single-junction structures previously discussed, using GaAs as the material. The general trend is that the optimal effective absorptance decreases with material quality. For a given material quality with $J_{A} / d<15 \mathrm{~A} \mathrm{~cm}^{-2} \mu \mathrm{m}^{-1}$, the optimal effective absorptance for Structure D is the largest, Structure C is the second largest, Structure A is the third largest, and Structure B is the smallest. While for a given material quality with $J_{A} / d>15 \mathrm{~A} \mathrm{~cm}^{-2} \mu \mathrm{m}^{-1}$, the optimal effective absorptance for Structure B is the largest, Structure $\mathrm{A}$ is the second largest, Structure $\mathrm{C}$ is the third largest, and Structure $\mathrm{D}$ is the smallest. Here, $J_{A} / d \approx 15 \mathrm{~A} \mathrm{~cm}^{-2} \mu \mathrm{m}^{-1}$ is a transition point where all four structures share the same optimal effective absorptance. As will be further analyzed, there is a transition from a radiative recombination dominated region to a $\mathrm{SRH}$ recombination dominated region between $1<J_{A} / d<15 \mathrm{~A} \mathrm{~cm}^{-2} \mu \mathrm{m}^{-1}$, which results in a

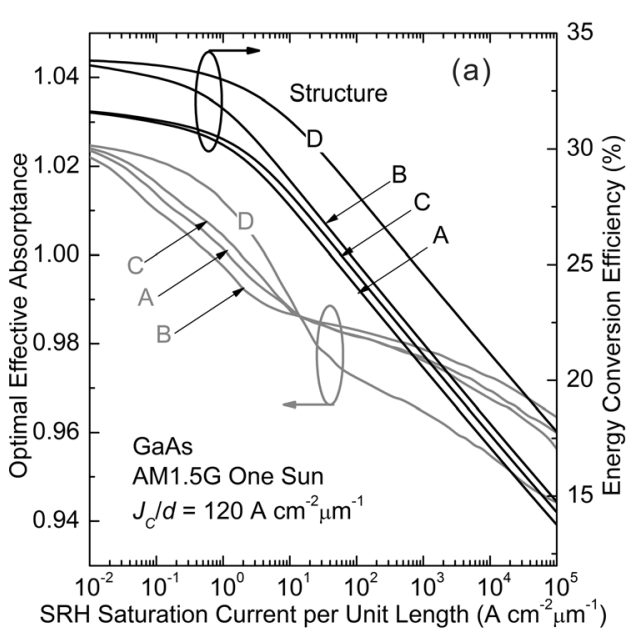

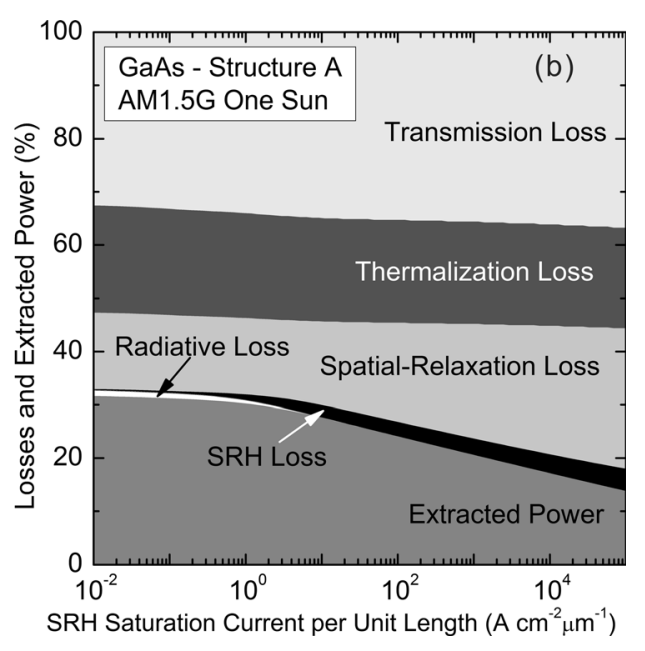

FIG. 11. Single-junction GaAs solar cell under one sun AM1.5 G with typical Auger recombination current density, $J_{C} / d=120 \mathrm{~A} \mathrm{~cm}^{-2} \mu \mathrm{m}^{-1}$. (a) Optimal effective absorptance (left-hand y-axis) and the corresponding conversion efficiency (right-hand y-axis) vs SRH recombination current density, $J_{A} / d$, for the four solar cell structures investigated (A, B, C, and D). (b) Solar power extracted and lost for Structure A at the optimal junction thickness vs SRH recombination current density, $J_{A} / d$. 

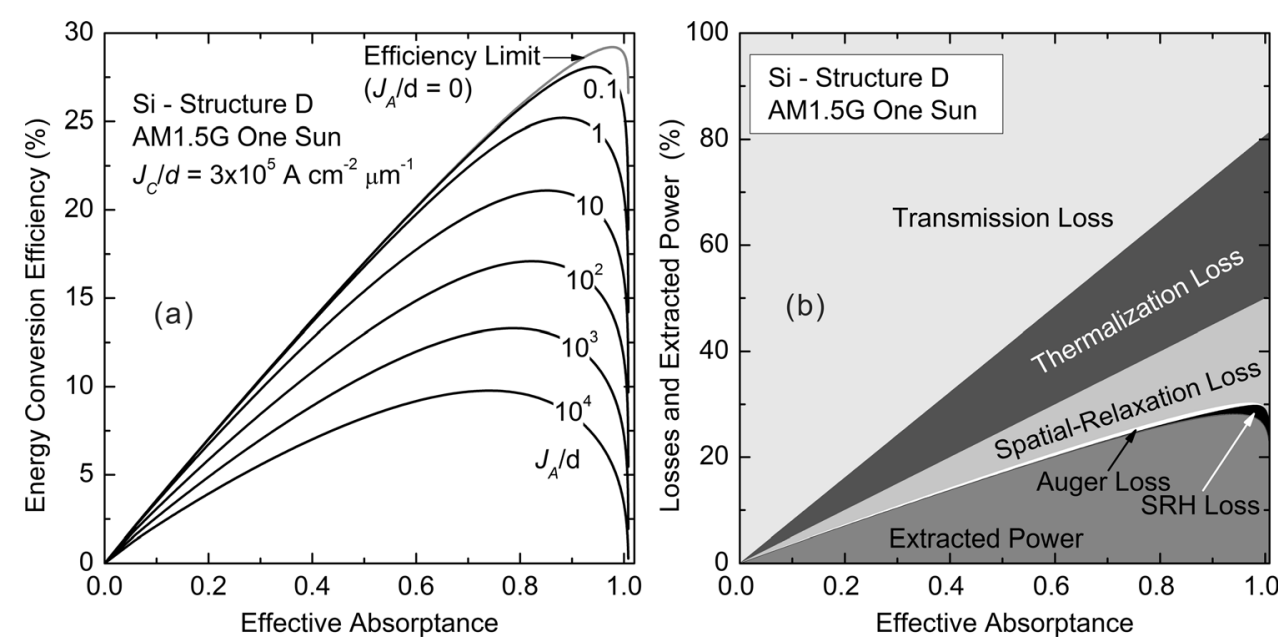

FIG. 12. Single-junction $\mathrm{Si}$ solar cell under one sun AM1.5 G with typical Auger recombination current density $J_{C} / d=3 \times 10^{5} \mathrm{~A} \mathrm{~cm}^{-2} \mu \mathrm{m}^{-1}$. (a) Efficiency vs junction absorptance (i.e., optical thickness) for various SRH recombination current densities, $J_{A} / d$. (b) Solar power extracted and lost vs absorptance for Structure D, assuming SRH recombination current density, $J_{A} / d=0.1 \mathrm{~A} \mathrm{~cm}^{-2} \mu \mathrm{m}^{-1}$. slope change in the optimal junction thickness curves shown in Fig. 11(a). Moreover, for a given material quality, the efficiency for Structure D is always the best; Structure B is next, Structure C is third, and Structure A is the lowest.

For very high quality materials $\left(J_{A} / d<10^{-2}\right.$ A cm ${ }^{-2} \mu \mathrm{m}^{-1}$ ) Structures D and B with reflecting back surfaces offer similar efficiencies at about $33.9 \%$, while Structures $\mathrm{C}$ and $\mathrm{A}$ on absorbing substrates offer similar and slightly lower efficiencies at about $31.7 \%$. In this case, the much larger radiative losses associated with an absorbing substrate results in a lower efficiency for Structures $\mathrm{C}$ and A. Moreover, the efficiency of the non-textured structures (A and B) approaches that of the textured structures (C and D) because the average optical path lengths related to the absorption of solar radiation and the emission loss are the same. On the contrary, as the material quality degrades, the textured top surface of Structures D and C provides a clear enhancement in performance as the radiative losses become insignificant and the nonradiative SRH losses take over. In this case, it is the thickness of the junction that becomes important, where thinner is better as long as the junction is thick enough to absorb most of the solar radiation; where, in order of performance, Structure D is the thinnest, Structure B is next, Structure C is next, and Structure A is the thickest.

To further understand these results, the extracted power and losses are plotted against the SRH saturation current density per unit length, $J_{A} / d$, in Fig. 11(b), for a GaAs based solar cell with an absorbing substrate (Structure A). Although the nonradiative recombination loss increases with the SRH recombination saturation current, the main contributor to the decrease in conversion efficiency is the large increase in the spatial-relaxation loss.

It is necessary to point out that state-of-the-art GaAs single-junction solar cells still operate in the SRH recombination dominated region, where $J_{A} / d$ varies from tens to hundreds of $\mathrm{Acm}^{-2} \mu \mathrm{m}^{-1}$. For Structure $\mathrm{A}$, the optimal junction thickness remains close to one that is optically-thick for $10^{-3}<J_{A} / d<10^{5} \mathrm{~A} \mathrm{~cm}^{-2} \mu \mathrm{m}^{-1}$, thus the transmission loss remains almost unchanged. On the contrary, for Structure $\mathrm{D}$ the optimal junction thickness deviates from one that is optically-thick and the transmission loss increases as the $\mathrm{SRH}$ recombination losses increase. For the best reported GaAs material quality $\left(J_{A} / d \approx 10 \mathrm{~A} \mathrm{~cm}^{-2} \mu \mathrm{m}^{-1}\right)$, the achievable efficiency limit under AM1.5 G one sun is $27.4 \%$ for Structure A and $30.8 \%$ for Structure D. In theory there could be a $2-3 \%$ absolute efficiency improvement from the current record if Structure D is adopted for GaAs singlejunction solar cells.

Similar results are plotted in Figs. 12(a), 12(b), 13(a), and 13(b) for single-junction Si solar cells. In the limiting case when SRH recombination is negligible, Si solar cells are dominated by Auger recombination (see the large Auger recombination saturation current in Fig. 9) due to a large effective density of states. Unlike direct bandgap materials, this results in a maximum in the efficiency limit curve (see Fig. 12(a)) that is a trade-off between the transmission and Auger recombination losses. For $J_{A} / d \approx 0.1 \mathrm{~A} \mathrm{~cm}^{-2} \mu \mathrm{m}^{-1}$ (see Fig. 12(b)), Auger recombination dominates the loss when the absorptance is less than 0.85 and SRH recombination dominates when the absorptance is greater than 0.85 .

As shown in Fig. 13(a), in the case when SRH recombination is negligible, the efficiency for non-textured front surfaces (Structures A and B) does not approach that of textured surfaces (Structures $\mathrm{C}$ and $\mathrm{D}$ ). Unlike radiative losses, Auger losses scale with the junction thickness and, hence, are different for each optimal thickness. As shown in Fig. 13(b), there is a transition from an Auger dominated region to an $\mathrm{SRH}$ dominated region between $0.1<J_{A} / d$ $<1 \mathrm{~A} / \mathrm{cm}^{2}$, which is about one order of magnitude smaller than that for GaAs. For the best reported $\mathrm{Si}$ material quality $\left(J_{A} / d \approx 0.5 \mathrm{~A} / \mathrm{cm}^{2}\right)$, the achievable efficiency limit under AM1.5 G one sun is $21.1 \%$ for Structure A and $26.4 \%$ for Structure D. Note that Structure D is ideal in that it assumes maximal random scattering at the textured top surface. The upper surface scattering for a real Si solar cell may not be entirely random, as is assumed in this study, which leads to thicker junction designs for optimal efficiency. ${ }^{25}$

Increasing the concentration of solar radiation is a successful approach to improve the conversion efficiency of solar cells ${ }^{18}$ and it is important to determine how the optimal design changes with solar concentration, which is presented in Figs. 14 and 15. In Fig. 14(a), the optimal junction absorptance (left-hand y-axis) and the corresponding conversion efficiency (right-hand y-axis) is plotted against solar concentration for single-junction GaAs solar cells for the four structures investigated. It can be seen that the optimal 

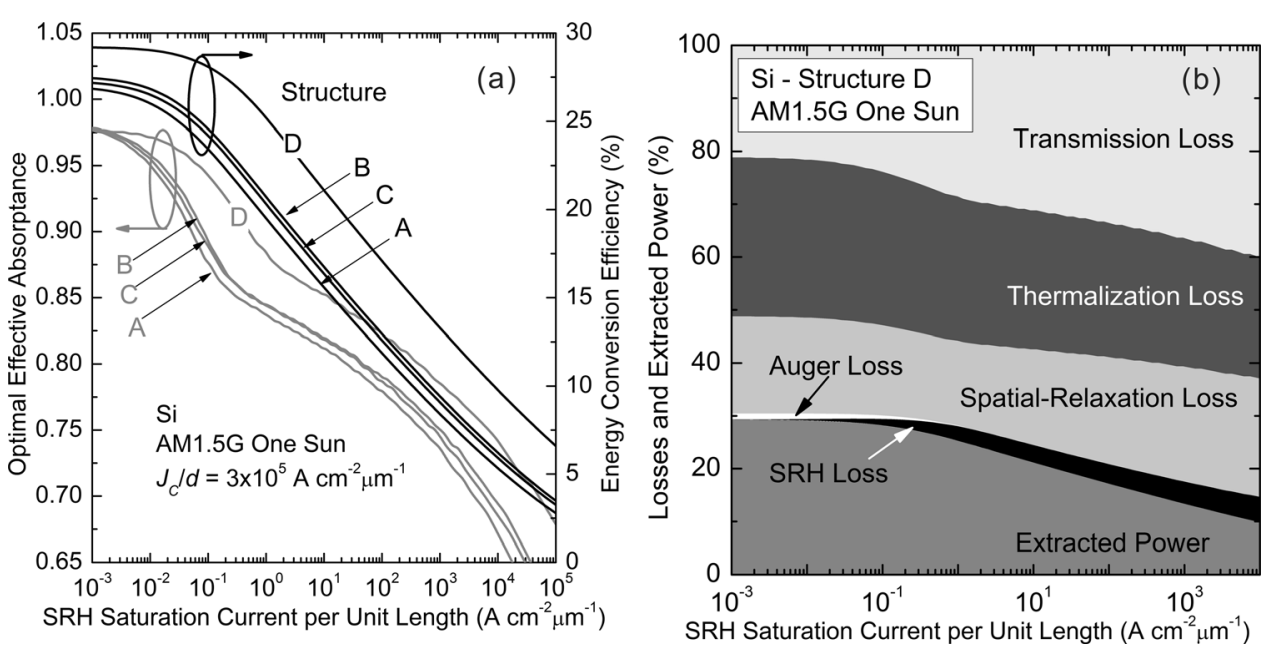

FIG. 13. Single-junction Si solar cell under one sun AM1.5 G with typical Auger recombination current density, $J_{C} / d=3 \times 10^{5} \mathrm{~A} \mathrm{~cm}^{-2} \mu \mathrm{m}^{-1}$. (a) Optimal effective absorptance (left-hand $y$ axis) and the corresponding conversion efficiency (right-hand $\mathrm{y}$-axis) vs SRH recombination current density, $J_{A} / d$, for the four solar cell structures investigated (A, B, C, and D). (b) Solar power extracted and lost for Structure D at the optimal junction thickness vs SRH current density, $J_{A} / d$. absorptance increases with solar concentration. For solar concentrations greater than one the effective absorptance of Structure D is the largest, Structure C is next, Structure A next, and Structure B is the smallest. The margin by which the textured surfaces outperform the non-textured surfaces decreases as the solar concentration increases.

In Fig. 14(b), the extracted power and losses are plotted against solar concentration for an optimally thick singlejunction GaAs solar cell with an absorbing substrate (Structure A). The efficiency monotonically increases with solar concentration since series resistance and other losses that increase with solar concentration are not included. As the solar concentration increases, the overall recombination loss slightly decreases, the spatial-relaxation losses substantially decreases, and the dominant recombination loss gradually changes from SRH to radiative. The main contribution of the concentration is that it increases the average free (extractable) energy of the photogenerated carriers.

Similar results are plotted in Figs. 15(a) and 15(b) for optimally thick single-junction $\mathrm{Si}$ solar cells with a textured front surface and reflecting back surface (Structure D). The change in optimal absorptance (junction thickness) with solar concentration is much greater for Si cells than GaAs cells. Moreover, the dominant recombination loss gradually changes from SRH to Auger for the Si cells. For solar concentrations less than 500 suns, the SRH recombination still dominates the total recombination loss for the $\mathrm{GaAs}$ and $\mathrm{Si}$ solar cells.

The preceding calculations and analysis provide a clear physical picture of single-junction devices and also deliver reasonable results compared to the actual devices. ${ }^{25,28}$

\section{B. Multi-junction solar cells}

To limit the number of possible combinations to model, the analysis of multi-junction solar cells assumes that the properties of each junction, other than bandgap energy, are based on prototypical GaAs, where the width of the absorption tail is $E_{u}=7 \mathrm{meV}$, the effective absorptance is $\overline{A_{\alpha}}=1$, the effective emittance from the upper surface is $\bar{\varepsilon}_{\text {upper }}=0.85$, the effective emittance of the lower surface is $\bar{\varepsilon}_{\text {lower }}=1.30$, and the Auger recombination loss is given by $J_{C}=380 \mathrm{~A} / \mathrm{cm}^{2}$. Note that the nonradiative losses are now expressed in terms of the junction property saturation current density, rather than the material property saturation current density per unit length used in the previous section. Nevertheless, in general, each parameter can be set independently for each junction in the model, provided specific material parameters are available.

Using a four-junction solar cell as an example, the overall energy conversion efficiency and the bandgaps of the junctions are calculated and plotted in Fig. 16(a), where $J_{A}$ is set
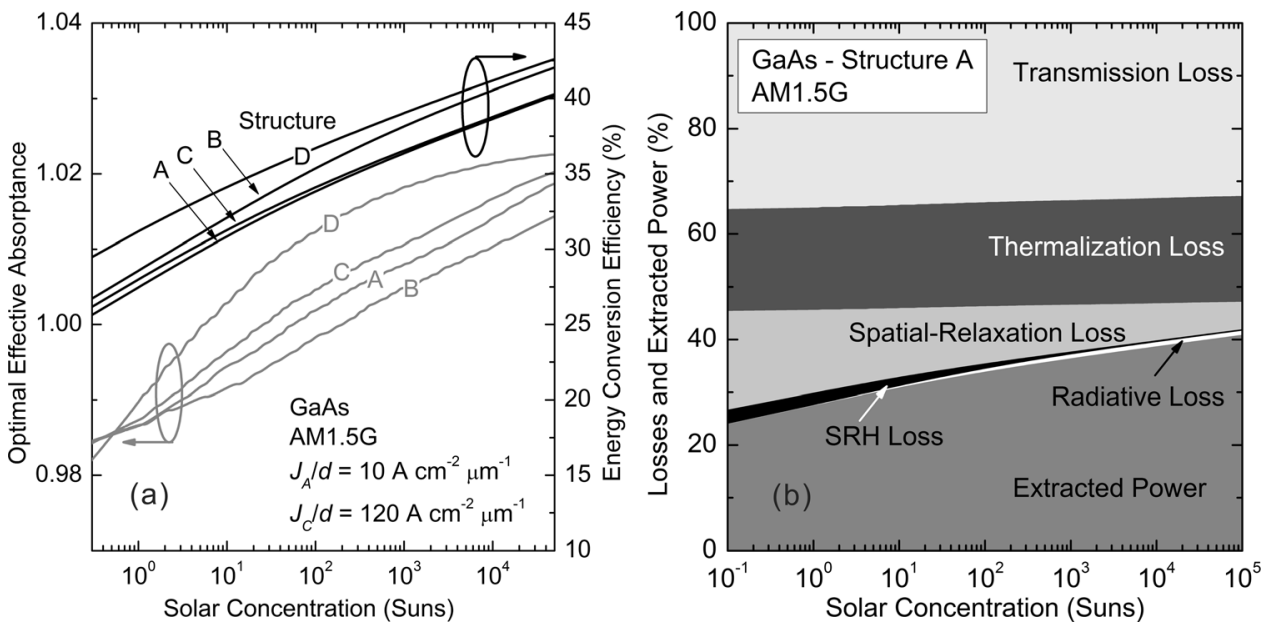

FIG. 14. Single-junction GaAs solar cell under AM1.5 G solar concentration with Auger recombination current density, $J_{C} / d=120 \mathrm{~A} \mathrm{~cm}^{-2} \mu \mathrm{m}^{-1}$ and $\mathrm{SRH}$ recombination current density, $J_{A} / d=10 \mathrm{~A} \mathrm{~cm}^{-2} \mu \mathrm{m}^{-1}$. (a) Optimal effective absorptance (left-band y-axis) and the corresponding conversion efficiency (right-hand y-axis) vs solar concentration for the four solar cell structures investigated (A, B, C, and D). (b) Solar power extracted and lost for Structure A at the optimal junction thickness vs solar concentration. 

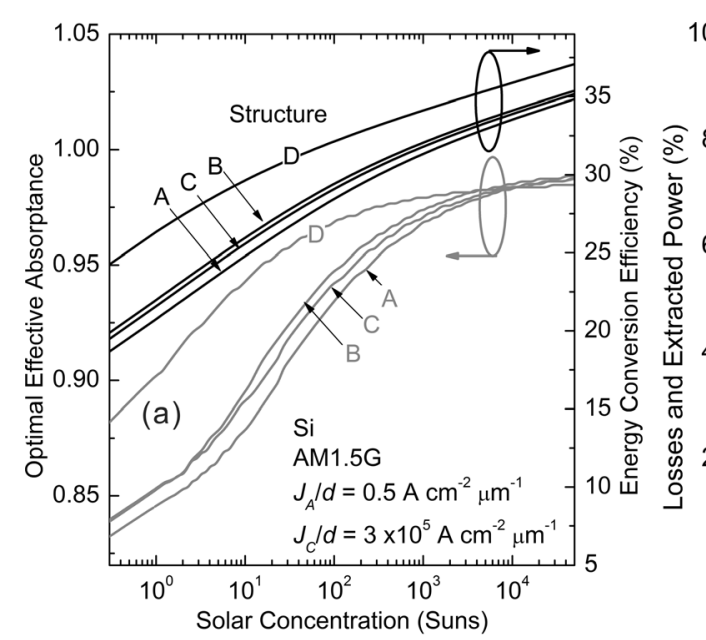

FIG. 15. Single-junction $\mathrm{Si}$ solar cell under AM1.5 G solar concentration with Auger recombination current density, $J_{C} / d=3 \times 10^{5} \mathrm{~A} \mathrm{~cm}^{-2} \mu \mathrm{m}^{-1}$ and SRH recombination current density, $J_{A} / d=0.5 \mathrm{~A} \mathrm{~cm}^{-2} \mu \mathrm{m}^{-1}$. (a) Optimal effective absorptance (left-hand y-axis) and the corresponding conversion efficiency (right-hand y-axis) vs solar concentration for the four solar cell structures investigated (A, B, C, and D). (b) Solar power extracted and lost for Structure D at the optimal junction thickness vs solar concentration. at $30 \mathrm{~A} / \mathrm{cm}^{2}$ (i.e., a reasonable estimate for state-of-the-art high-quality material) and spontaneous emission coupling is taken into account. To further identify the trend of each loss mechanism versus the top-junction bandgap, the extracted power and losses versus the bandgap energy of the top junction are plotted in Fig. 16(b). In Fig. 16(a), there are two efficiency peaks at $\sim 44 \%$ when the bandgaps of the top junction are 2.02 and $2.10 \mathrm{eV}$. This double peak characteristic is mainly due to the atmospheric absorption features in the AM1.5 G spectrum. Any increase (or decrease) of the top junction bandgap apart from these maximum points will result in a lower conversion efficiency due to an increase in the total loss. Moreover, the energy separation between bandgaps increases as the top junction bandgap decreases and the bandgap energy separation is smaller for the bottom junctions. Furthermore, the bottom junction cannot provide sufficient current to satisfy the current matching condition when the top junction bandgap is below $2.01 \mathrm{eV}$ under one sun condition.

To further understand the impact of SRH recombination on multi-junction solar cells, the extracted power and losses and the maximum conversion efficiency and corresponding bandgap of each junction as a function of the SRH recombination saturation current density, $J_{A}$, are modeled. The results for four-junction solar cells are shown in Fig. 17(a) for the maximum conversion efficiency and bandgap and in Fig. 17(b) for the extracted power and losses. Here, the SRH recombination saturation current density, $J_{A}$, is the same for each junction to simplify the discussion, without a loss in generality since the overall results remain the same if the values differ between junctions.

When $J_{A}<1 \mathrm{~A} / \mathrm{cm}^{2}$ the energy conversion efficiency is very close to the theoretical limit and the recombination loss is dominated by the radiative recombination. As $J_{A}$ increases, the SRH nonradiative recombination gradually displaces the radiative recombination. For $J_{A}>1 \mathrm{~A} / \mathrm{cm}^{2}$, there is a substantial increase of the spatial-relaxation loss, as discussed in Sec. II and the optimal bandgaps for all the junctions deviate from those for ideal materials, resulting in greater transmission losses and a slightly smaller thermalization loss due to the smaller energy spacing between the bandgaps of adjacent junctions. Clearly, the total recombination loss is dominated by the SRH recombination in state-ofthe-art multi-junction solar cells under one sun when $J_{A}$ varies from tens to hundreds of $\mathrm{A} / \mathrm{cm}^{2}$.

The maximum efficiency and the corresponding bandgap energies versus solar concentration for fourjunction solar cells are shown in Fig. 18(a) and the extracted power and losses versus solar concentration are shown in Fig. 18(b). Figure 18(a) shows that the peak efficiency increases as the optimum bandgap energies for the junctions decrease with the solar concentration. Figure 18(b) shows that such an efficiency increase is mainly due to the
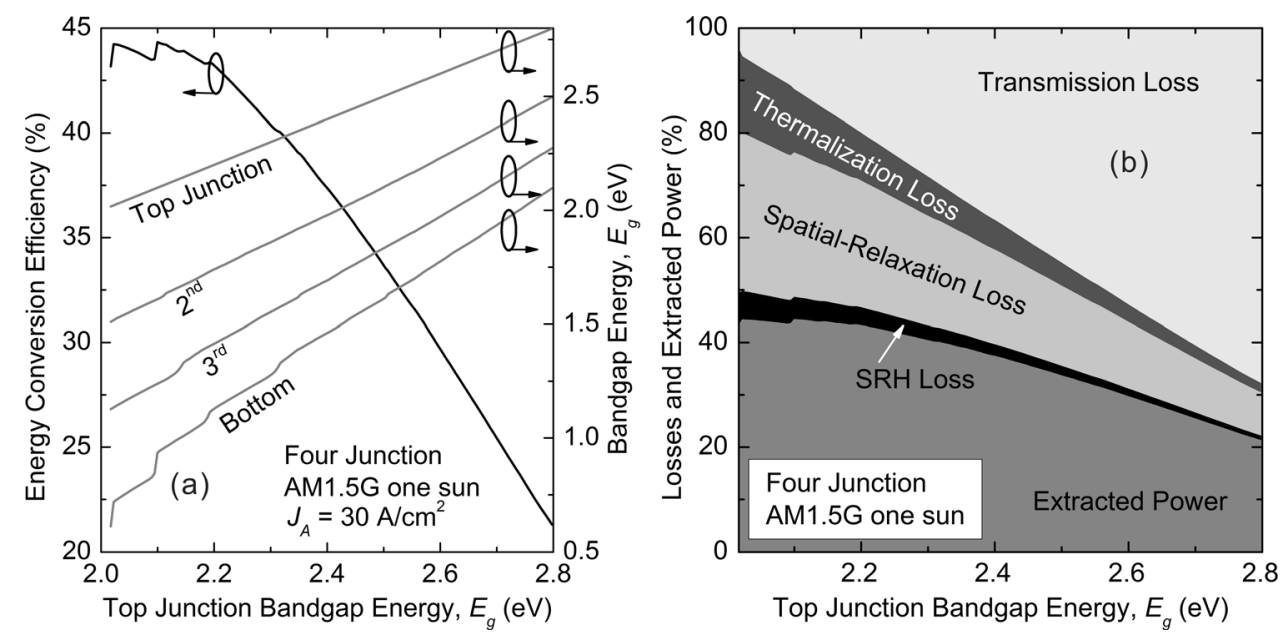

FIG. 16. Efficiency of four-junction solar cell vs top-junction bandgap energy with SRH recombination current density, $J_{A}=30 \mathrm{~A} / \mathrm{cm}^{2}$. (a) Power conversion efficiency (left-hand y-axis) and bandgap energy of each junction (right-hand y-axis). (b) Solar power extracted and lost (y-axis). 

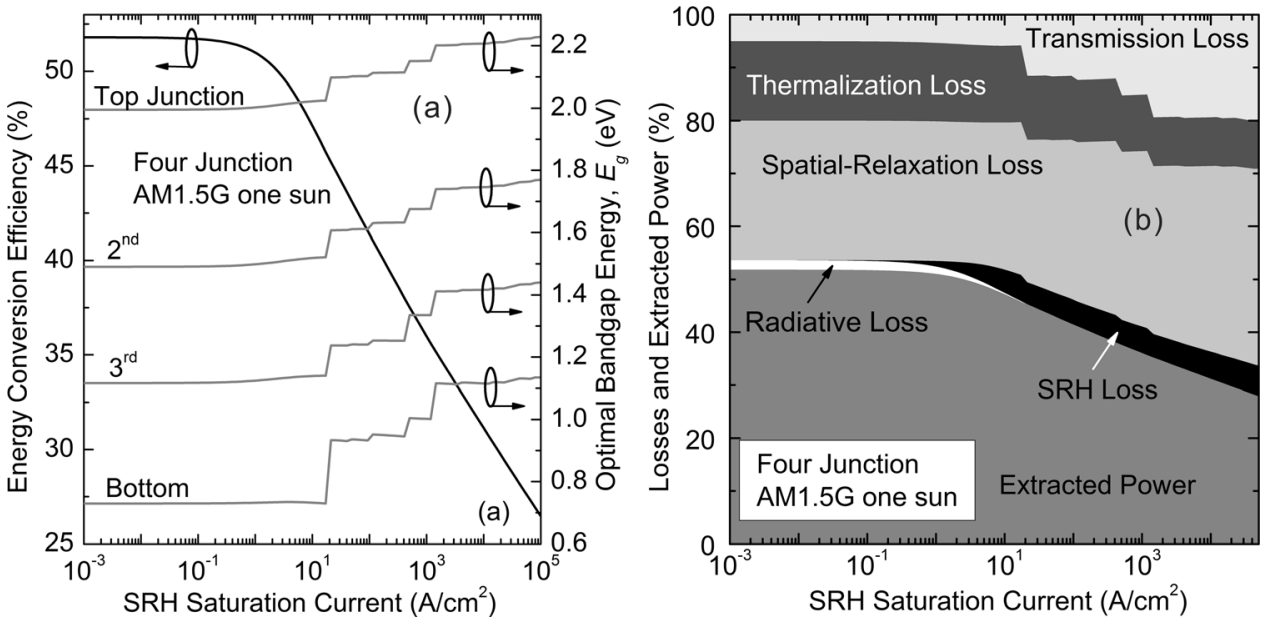

FIG. 17. Efficiency of four-junction solar cell vs SRH recombination current density, $J_{A}$. (a) Power conversion efficiency (left-hand y-axis) and optimal bandgap energy for each junction (righthand y-axis). (b) Solar power extracted and lost (y-axis). reduction in transmission, spatial-relaxation, and the SRH recombination losses. It is interesting to point out that the thermalization loss increases with concentration as the optimal junction bandgaps decrease with concentration. Moreover, the radiative recombination loss gradually increases in terms of the overall recombination loss. For the solar concentration less than 500 suns, SRH recombination still dominates in the total recombination loss. These findings are important for multi-junction solar cell designs because they show that the bandgaps need to be carefully optimized, taking into account material quality and solar concentration.

It is interesting to further investigate how much improvement in efficiency can be achieved by utilizing more junctions. The peak efficiency versus the junction number is shown in Fig. 19(a) for various values of $J_{A}$, while the extracted power and losses versus the junction number are shown in Fig. 19(b) for $J_{A}=30 \mathrm{~A} / \mathrm{cm}^{2}$. A net increase in the conversion efficiency is achieved in solar cell designs with a larger number of junctions because the reduction of transmission and thermalization losses more than offsets the increase in the spatial-relaxation and SRH recombination losses caused by the decrease in the injection level of each junction. A careful examination of Fig. 19(a) further reveals that the benefit of having more junctions gradually disappears as the material quality is reduced.
In all of the preceding calculations, the AM1.5 G solar spectrum is used. If the AM0 (Ref. 26) spectrum is used, the principles remain the same, but the results will be different. In general, the calculated curves for AM0 are much smoother than that for AM1.5 G.

This work extends existing detailed balance models for solar cells by considering the impact on performance of: i) SRH and Auger recombination, ii) optical design in terms of light trapping, absorption, and reflection, iii) less than ideal photon recycling, and iv) the non-ideal absorption of solar radiation and the spontaneous emission by tail states. Although it is possible to further incorporate more extrinsic loss mechanisms, such as surface recombination and resistance, these extrinsic mechanisms are device structure dependent and are not compatible with this generic model that only takes into account the fundamental parameters of bulk materials. In general, this work provides the achievable limits for solar cells made of practical materials and provides clear device design principles and the related underlying physics. Although not presented here, more practical design principles can be accessed using this model, such as optimal junction thicknesses of all junctions in a multi-junction cell with non-ideal bandgaps that are not current matched in the optically-thick limit.

It is necessary to point out that it takes only a few hours to calculate all of the preceding diagrams using a personal
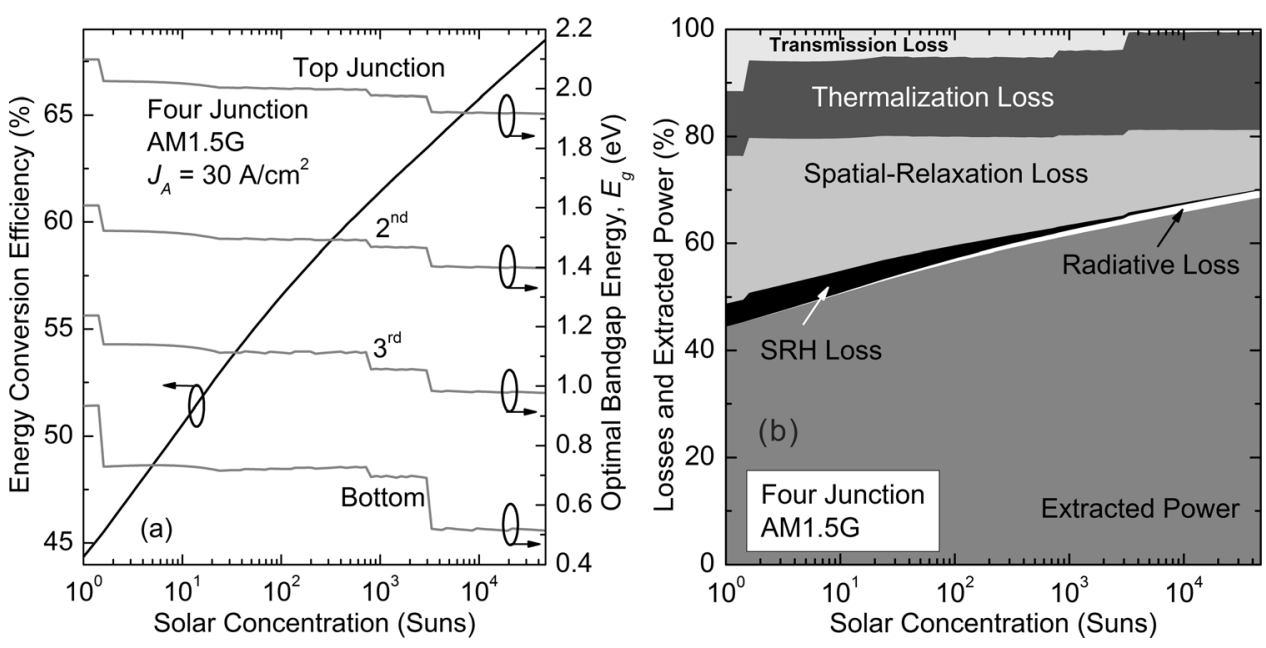

FIG. 18. Efficiency of four-junction solar cell vs AM1.5 G solar concentration with SRH recombination current density, $J_{A}=30 \mathrm{~A} / \mathrm{cm}^{2}$. (a) Power conversion efficiency (left-hand y-axis) and optimal bandgap energy for each junction (righthand y-axis). (b) Solar power extracted and lost (y-axis). 

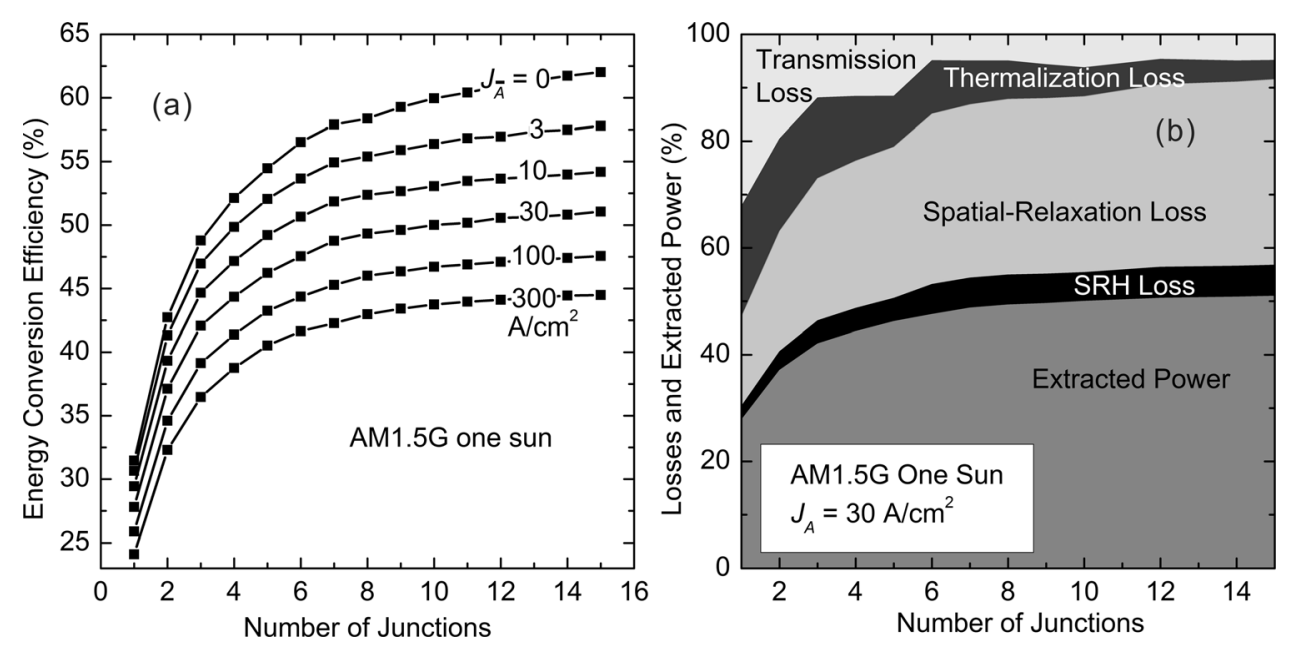

FIG. 19. Solar cell efficiency vs number of junctions. (a) Optimal efficiency for various SRH recombination current densities, $J_{A}$. (b) Solar power extracted and lost for $J_{A}=30 \mathrm{~A} / \mathrm{cm}^{2}$. computer. Normally, the calculation time is much longer if sophisticated numerical simulation tools are used to reproduce these diagrams. It is known that the complexity of the solar cell design dramatically increases with the junction number since junctions are made of dissimilar materials with different material parameters. For a given practical multijunction solar cell design, the optimization processes are time-consuming because the necessary current-matching requirement requires a large number of iterations to solve the drift and diffusion equations for all of the junctions to find the optimal bandgap energies and layer thicknesses. ${ }^{30}$ In this regard, this model offers a powerful approach to gathering initial device design parameters such as bandgap and layer thicknesses. In addition, the principles and equations used in this study are significant for other optoelectronic devices. For example, the equations used to calculate emittance are suitable for LED device design without the use of complex ray-tracing simulations.

\section{CONCLUSIONS}

A semi-analytical model for photovoltaic singlejunction and multi-junction solar cells is established that provides insight into the intricate workings of ideal and practical solar cells by taking into account the impact of photon recycling, spontaneous emission coupling, non-radiative recombination, and non-ideal step-like absorptance and emittance due to the presence of tail states in real materials. Four types of fundamental losses for solar cells are discussed, including transmission, thermalization, spatial-relaxation, and recombination. To quantitatively analyze these losses, the average absorptance and emittance are defined and their trends versus junction thickness are determined for single-junction GaAs and Si solar cells for four different structures with: i) a smooth top surface and an absorbing substrate which typifies a subcell within a multi-junction solar cell, ii) a smooth top surface and a reflecting substrate (i.e., a reflecting back surface), iii) a textured top surface and an absorbing substrate, and iv) a textured top surface and a reflecting substrate which typifies a high performance single-junction solar cell.

The contribution of the width of the Urbach tail is found to be significant to the overall emission since it is on the order of the width of the spontaneous emission spectrum, while it is insignificant to the overall absorption of solar radiation since it is small compared to the width of the solar spectrum. The SRH, radiative, and Auger recombination saturation currents $\left(J_{A}, J_{B}\right.$, and $\left.J_{C}\right)$ are analyzed based upon published material parameters for several common semiconductors. The SRH recombination saturation current density per unit length, $J_{A} / d$, for the best reported material quality varies from 0.03 to $300 \mathrm{~A} \mathrm{~cm}^{-2} \mu \mathrm{m}^{-1}$ for various group IV and III-V semiconductors and has no clear trend versus the bandgap energy. The radiative recombination saturation current density, $J_{B}$, increases monotonically with the bandgap energy and is substantially lower for structures with reflecting back surfaces compared to that for structures with absorbing substrates. The Si has the largest Auger recombination saturation current density per unit length due to its large effective densities of states.

The optimal thickness of single-junction solar cells decreases as the material quality decreases due to a trade-off between transmission and SRH recombination losses. For both GaAs and Si solar cells using the best reported material quality, SRH is the dominant bulk recombination loss. Moreover, whenever the SRH saturation current is greater than $10 \mathrm{~A} / \mathrm{cm}^{2}$, SRH recombination dominates, and whenever the $\mathrm{SRH}$ saturation current is less than $1 \mathrm{~A} / \mathrm{cm}^{2}$, radiative recombination dominates for GaAs and Auger recombination dominates for $\mathrm{Si}$.

The energy conversion efficiency and optimal junction thickness increases with the solar concentration. The efficiency improvement is mainly due to a reduction in spatialrelaxation. As the solar concentration increases from 100 to 1000 suns, there is a transition in the dominant loss mechanism from the SRH to radiative in GaAs and the SRH to $\mathrm{Au}-$ ger in Si. Moreover, in multi-junction solar cells, as the solar concentration increases the optimal bandgaps decrease and as the SRH recombination current density increases, the optimal bandgaps increase. Furthermore, the efficiency increase with junction number is due to a substantial decrease in the transmission and thermalization losses. However, since the spatial-relaxation and SRH recombination losses increase with the junction number, the efficiency gain eventually saturates as the diminishing reduction in the transmission and thermalization losses approaches the increase in the spatialrelaxation and SRH losses. For a four junction solar cell 
structure, the optimal top-junction bandgap is 2.02 or 2.10 $\mathrm{eV}$, which results in an efficiency of around $44 \%$ under AM1.5 G one sun.

\section{ACKNOWLEDGMENTS}

This work is supported by the Science Foundation Arizona Contract Nos. SRG 0190-07 and SRG 0339-08, the Air Force Research Laboratories/Space Vehicles Directorate Contract No. FA9453-08-2-0228, and the National Science Foundation Grant No. 1002114. The authors thank Swee H. Lim, Shi Liu, Jing-Jing Li, and Shuming Wang for valuable discussions on some of the topics in this paper.

${ }^{1}$ S. M. Sze, Physics of Semiconductor Devices (Wiley, New York, 1981).

${ }^{2}$ W. Shockley and H. J. Queisser, J. Appl. Phys. 32, 510 (1961).

${ }^{3}$ C. H. Henry, J. Appl. Phys. 51, 4494 (1980).

${ }^{4}$ T. Tiedje, E. Yablonovitch, G. D. Cody, and B. G. Brooks, IEEE Trans. Electron Devices ED-31, 711 (1984).

${ }^{5}$ G. L. Araujo and A. Marti, IEEE Trans. Electron Devices 37, 1402 (1990).

${ }^{6}$ S.-L. Chuang, Physics of Photonic Devices, 2nd Ed. (Wiley, New Jersey, 2009).

${ }^{7}$ F. Stern and J. M. Woodall, J. Appl. Phys. 45, 3904 (1974).

${ }^{8}$ J.-B. Wang, D. Ding, S. R. Johnson, S.-Q. Yu, and Y.-H. Zhang, Phys. Status Solidi B 244, 2740 (2007).

${ }^{9}$ A. Marti and G. L. Araujo, Sol. Energy Mater. Sol. Cells 43, 203 (1996).

${ }^{10}$ C. Baur, M. Hermle, F. Dimroth, and A. W. Bett, Appl. Phys. Lett. 90, 192109 (2007).

${ }^{11}$ S. H. Lim, J.-J. Li, E. H. Steenbergen, and Y.-H. Zhang, "Luminescence coupling effects on multi-junction solar cell external quantum efficiency measurement, Progress in Photovoltaics: Research and Applications," DOI: 10.1002/pip.1215, published on line Nov 28 (2011).
${ }^{12}$ J.-J. Li, S. H. Lim, C. R. Allen, D. Ding, and Y.-H. Zhang, "Combined effects of shunt and luminescence coupling on external quantum efficiency measurement of multijunction solar cells," IEEE Journal of Photovoltaics, DOI: 10.1109/JPHOTOV.2011.2172188, published on line Nov 28 (2011).

${ }^{13}$ D. Ding, S. R. Johnson, and Y.-H. Zhang, in Proceedings of the 35th IEEE Photovoltaic Specialists Conference (PVSC), Hawaii, 18-25 June 2010).

${ }^{14}$ L. Lockhartand L. R. King, J. Opt. Soc. Am. 37, 689 (1947).

${ }^{15}$ J. Rodriguez, I. Tobias, and A. Luque, Sol. Energy Mater. Sol. Cells 45, 241 (1997).

${ }^{16}$ R. G. Gordon, MRS Bull. 25, 52 (2000).

${ }^{17}$ P. J. Verlinden, R. A. Sinton, K. Wickham, R. A. Crane, and R. M. Swanson, Proceedings of the 14th European Photovoltaic Solar Energy Conference, Barcelona, Spain, 30 June - 4 July 1997, p. 96.

${ }^{18}$ A. Luque and S. Hegedus, Handbook of Photovoltaic Science and Engineering (Wiley, New Jersey, 2003).

${ }^{19} \mathrm{http}: / /$ rredc.nrel.gov/solar/spectra/am1.5/, see spectra download links.

${ }^{20}$ S. R. Johnson and T. Tiedje, J. Appl. Phys. 78, 5609 (1995).

${ }^{21}$ D. Ding, "Thermodynamic analysis and determination of spontaneous emission quantum efficiency for luminescence refrigeration in semiconductors," Ph.D. dissertation, Arizona State University, 2007.

${ }^{22}$ C. H. Grein and S. John, Phys. Rev. B 39, 1140 (1989).

${ }^{23}$ M. Beaudoin, A. J. G. DeVries, S. R. Johnson, H. Laman, and T. Tiedje, Appl. Phys. Lett. 70, 3540 (1997)

${ }^{24}$ S. R. Johnson, D. Ding, J.-B. Wang, S.-Q. Yu, and Y.-H. Zhang, J. Vac. Sci. Technol. B 25, 1077 (2007).

${ }^{25}$ J. Zhao, A. Wang, and M. A. Green, Solar Energy Mater. Solar Cells 66, 27 (2001).

${ }^{26} \mathrm{http}: / /$ rredc.nrel.gov/solar/spectra/am0/, see spectra download links

${ }^{27}$ E. D. Palik, Handbook of Optical Constants of Solids (Academic, New York, 1985).

${ }^{28}$ G. J. Bauhuis, P. Mulder, J. J. Schermer, E. J. Haverkamp, J. van Deelen, and P. K. Larsen, Proceedings of 20th European Photovoltaic Solar Energy Conference, Barcelona, Spain, June 6-10, 2005, p. 468.

${ }^{29} \mathrm{~S}$. Adachi, Properties of Group-IV, III-V and II-VI (John Wiley \& Sons, Hoboken, NJ, 2005).

${ }^{30}$ S.-N. Wu, D. Ding, S. R. Johnson, S.-Q. Yu, and Y.-H. Zhang, Prog. Photovoltaics 18, 328 (2010). 